Article

\title{
Chemo-selective couplings of anilines and acroleins/enones under substrate control and condition control
}

\author{
Xukai Zhou a,b, Jiaqiong Sun a, Xingwei Li a,* \\ a Dalian Institute of Chemical Physics, Chinese Academy of Sciences, Dalian 116023, Liaoning, China \\ b University of Chinese Academy of Sciences, Beijing 100049, China
}

\section{A R T I C L E I N F O}

\section{Article history:}

Received 29 June 2018

Accepted 5 July 2018

Published 5 November 2018

\section{Keywords:}

$\mathrm{Rh}(\mathrm{III})$-catalysis

Chemo-selectivity

Annulation

Substrate control

Condition control

\begin{abstract}
A B S T R A C T
$\mathrm{Rh}(\mathrm{III})$-catalyzed $\mathrm{C}-\mathrm{H}$ activation of $\mathrm{N}$-protected anilines and chemo-divergent couplings with acroleins/enones have been realized for synthesis of three classes of heterocycles. The oxidative coupling of $N$-pyridylaniline afforded dihydroquinolones with the acrolein being a major hydrogen acceptor. When the directing group was replaced by pyrimidyl in the same system, redox-neutral coupling occurred to afford hemiaminal ethers. Oxidative annulation of $N$-pyridylanilines with enones using $\mathrm{AgBF}_{4}$ oxidant afforded atropisomeric quinolinium salts.
\end{abstract}

(C) 2018, Dalian Institute of Chemical Physics, Chinese Academy of Sciences. Published by Elsevier B.V. All rights reserved.

\section{Introduction}

Metal-catalyzed activation of $\mathrm{C}-\mathrm{H}$ bonds has allowed development of numerous efficient approaches to access various value-added organics, especially heterocycles [1-11]. In $\mathrm{C}-\mathrm{H}$ activation chemistry, chemo-, regio-, or stereoselectivity of $\mathrm{C}-\mathrm{H}$ activation constitutes a central challenge in that environmentally analogous $\mathrm{C}-\mathrm{H}$ bonds are generally present. Consequently, controlling selectivity of $\mathrm{C}-\mathrm{H}$ activation has received increasing attention [12-18]. Despite the significant progress, regulation of redox-selectivity has been less studied [19-21].

Organic redox reactions, classically defined as gaining/losing hydrogen/oxygen, are ubiquitous. The transfer of a hydrogen atom in the form of a hydride, radical, or proton contributes to redox-diversity [22-25]. For example, elimination of a hydride from organics leads to oxidation, while proton trans- fer retains the oxidation state. Regulating the redox-chemistry of catalytic reactions represents an important task, and ideally all the three oxidation states (oxidation, reduction, and redox-neutrality) are selectively attained. However, related systems have not been described to the best of our knowledge.

$\mathrm{Cp} * \mathrm{Rh}(\mathrm{III})$-catalyzed arene $\mathrm{C}-\mathrm{H}$ activation followed by cyclization has served as a cornerstone for synthesizing cyclic structures [26-33]. The $\mathrm{C}-\mathrm{H}$ activation of anilines has been well-explored for synthesis of heterocycles using unsaturated coupling partners [34-37]. We recently reported the integration of $\mathrm{C}-\mathrm{H}$ activation and transfer hydrogenation (TH) in the coupling of anilines and enones under Ir(III) and Rh(III) catalysis, which afforded two reductive products (E, F) and one redox-neutral product (D, Scheme 1) [38]. We reasoned that the TH reduction can be extended to TH oxidation [39-41] or external oxidation. During our investigations on the coupling

\footnotetext{
* Corresponding author. Tel: +86-411-84379089; E-mail: xwli@dicp.ac.cn This work was supported by the National Nature Science Foundation of China $(21525208,21472186)$ and the fund for new technology of methanol conversion of Dalian Institute of Chemical Physics (Chinese Academy of Sciences).

DOI: 10.1016/S1872-2067(18)63134-1 | http://www.sciencedirect.com/science/journal/18722067 | Chin. J. Catal., Vol. 39, No. 11, November 2018
} 

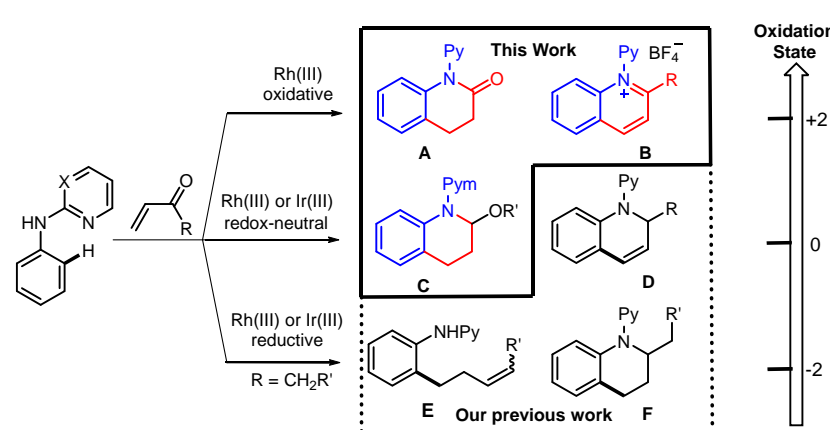

Scheme 1. Redox-switch in the coupling of anilines and acroleins/enones.

between the same anilines and acroleins or enones, we realized oxidative synthesis of two heterocycles, namely dihydroquinolones (A) and quinolinium salts (B). In addition, in the case of acrolein coupling partner, hemiaminal ether (C) was obtained via redox-neutral coupling in an alcoholic solvent (Scheme 1).

The $\mathrm{C}-\mathrm{H}$ activation of anilines en route to hydroarylation of acrolein/enones and nucleophilic cyclization generates a $\mathrm{Rh}$ (III) alkoxide intermediate (G) which is a common intermediate for further transformations (Scheme 2). In the case of an acrolein, $\beta$-hydrogen elimination of $\mathbf{G}$ is proposed to furnish a dihydroquinolone (A) together with formation of a $\mathrm{Cp} * \mathrm{RhXH}$, which is a direct precursor of $\mathrm{Cp} * \mathrm{Rh}(\mathrm{I})$ intermediate that can be reoxidized by an external oxidant or by the acrolein (via transfer hydrogenation). In fact, this $\beta$-hydrogen elimination has been realized for amide/lactam synthesis in related Rh(III)-catalyzed reactions of aldehydes [42-44]. Alternatively, the alkoxide intermediate $\mathbf{G}$ may undergo protonolysis and elimination of water to afford an iminium species that is prone to $\mathrm{TH}$ reduction [45] or nucleophilic addition. We now report these divergent couplings under substrate/condition control.

\section{Experimental}

\subsection{General}

All chemicals were obtained from commercial sources and were used as received unless otherwise noted. $N$-pyridylaniline [46], $N$-pyrimidylindole [47], and $2 \mathbf{e}$ [48], were prepared by following the literature reports. All reactions were carried out using Schlenk techniques or in a nitrogen-filled glove box. ${ }^{1} \mathrm{H}$

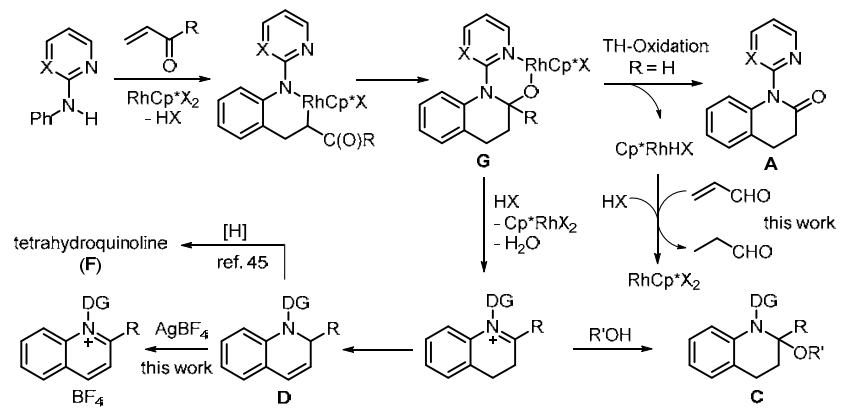

Scheme 2. Diverse pathways of the coupling of anilines and acroleins/enones. and ${ }^{13} \mathrm{C}$ NMR spectra were recorded using $\mathrm{CDCl}_{3}$ as a solvent on a bucker $400 \mathrm{MHz}$ NMR spectrometer. The chemical shift is given in dimensionless $\delta$ values and is referenced relative to TMS in ${ }^{1} \mathrm{H}$ and ${ }^{13} \mathrm{C}$ NMR spectroscopy. All coupling constants $($ ) were reported in Hertz (Hz). Multiplicities are reported as follows: singlet (s), doublet (d), doublet of doublets (dd), triplet $(\mathrm{t})$, quartet $(\mathrm{q})$, and multiplet $(\mathrm{m})$. HRMS data were obtained via ESI mode with a TOF mass analyzer. Column chromatography was performed on silica gel (300-400 mesh) with freshly distilled ethyl acetate (EA) and petroleum ether (PE).

\subsection{General procedure for the synthesis of compounds $3,4,6$}

$N$-(2-pyrimidyl)anilines (1, $0.2 \mathrm{mmol}), \alpha, \beta$-unsaturated ketones (2, $0.4 \mathrm{mmol}),\left[\mathrm{Cp}^{*} \mathrm{RhCl}_{2}\right]_{2}$ ( $\left.4 \mathrm{~mol} \%\right), \mathrm{AgBF}_{4}$ (2.5 equiv), $\mathrm{MeOH}(3.0 \mathrm{~mL})$ were charged into a Schlenk tube under a nitrogen atmosphere. The reaction mixture was stirred at $120{ }^{\circ} \mathrm{C}$ for $12 \mathrm{~h}$. Then the solvent was removed under reduced pressure, and the residue was purified by silica gel chromatography using DCM/MeOH to afford compound 3.

$N$-(2-pyridyl)anilines (1, $0.2 \mathrm{mmol})$, acrolein (2f, $0.4 \mathrm{mmol}$ ), [Cp* $\left.\mathrm{RhCl}_{2}\right]_{2}$ (5 mol\%), $\mathrm{AgSbF}_{6}$ (20 mol\%), HOAc (2 equiv), $\mathrm{Cu}(\mathrm{OAc})_{2}(0.3$ equiv), and acetone $(3.0 \mathrm{~mL})$ were charged into a Schlenk tube under a nitrogen atmosphere. The reaction mixture was stirred at $40{ }^{\circ} \mathrm{C}$ for $12 \mathrm{~h}$. Then the solvent was removed under reduced pressure, and the residue was purified by silica gel chromatography using EA/PE to afford compound 4.

$\mathrm{N}$-(2-pyrimidyl)anilines (5, $0.2 \mathrm{mmol})$, acrolein (2f, 0.4 mmol), $\left[\mathrm{Cp}^{*} \mathrm{RhCl}_{2}\right]_{2}$ (2.5 mol\%), AgSbF 6 (10 mol\%), HOAc (2 equiv), $\mathrm{Ni}(\mathrm{OAc})_{2} \cdot 4 \mathrm{H}_{2} \mathrm{O}(30 \mathrm{~mol} \%)$ and $\mathrm{EtOH}(3.0 \mathrm{~mL})$ were charged into a Schlenk tube under a nitrogen atmosphere. The reaction mixture was stirred at $40{ }^{\circ} \mathrm{C}$ for $12 \mathrm{~h}$. Then the solvent was removed under reduced pressure, and the residue was purified by silica gel chromatography using EA/PE to afford compound 6.

\subsection{Spectral data for products}

3aa, brown solid (54.5 mg, 85\% yield). ${ }^{1} \mathrm{H}$ NMR (400 MHz, $\left.\mathrm{CDCl}_{3}\right): \delta=9.16(\mathrm{~d}, J=8.7 \mathrm{~Hz}, 1 \mathrm{H}), 8.81(\mathrm{~d}, J=3.6 \mathrm{~Hz}, 1 \mathrm{H})$, 8.35-8.24 (m, 2H), 8.12 (d, $J=8.7 \mathrm{~Hz}, 1 \mathrm{H}), 8.00(\mathrm{~d}, J=7.9 \mathrm{~Hz}$, $1 \mathrm{H}), 7.95-7.78(\mathrm{~m}, 3 \mathrm{H}), 7.07$ (d, $J=8.7 \mathrm{~Hz}, 1 \mathrm{H}), 3.10-2.99(\mathrm{~m}$, 1H), 2.83-2.72 (m, 1H), $1.38(\mathrm{t}, J=7.5 \mathrm{~Hz}, 3 \mathrm{H}) .{ }^{13} \mathrm{C}$ NMR $(100$ $\left.\mathrm{MHz}, \mathrm{CDCl}_{3}\right): \delta=165.0,151.0,149.8,148.6,141.5,139.8,135.6$, 130.5, 129.7, 128.2, 127.4, 123.5, 123.0, 119.4, 28.9, 12.6 . HRMS: $\left[\mathrm{M}-\mathrm{BF}_{4}\right]^{+}$calculated for $\mathrm{C}_{16} \mathrm{H}_{15} \mathrm{~N}_{2}$ 235.1230, found 235.1230 .

3ab, yellow solid (56.4 mg, 78\% yield). ${ }^{1} \mathrm{H}$ NMR (400 MHz, $\left.\mathrm{CDCl}_{3}\right): \delta=9.19(\mathrm{~d}, J=8.6 \mathrm{~Hz}, 1 \mathrm{H}), 8.83(\mathrm{~d}, J=3.9 \mathrm{~Hz}, 1 \mathrm{H})$, 8.35-8.29 (m, 2H), 8.10 (d, $J=8.6 \mathrm{~Hz}, 1 \mathrm{H}), 7.98-7.90(\mathrm{~m}, 2 \mathrm{H})$, 7.88-7.83 (m, 2H), 7.08 (d, $J=8.7 \mathrm{~Hz}, 1 \mathrm{H}), 3.06-2.97(\mathrm{~m}, 1 \mathrm{H})$, 2.75-2.66 (m, 1H), 1.81-1.72 (m, 2H), 1.27-1.18 (m, 4H), 0.81 $(\mathrm{t}, J=6.9 \mathrm{~Hz}, 3 \mathrm{H}) .{ }^{13} \mathrm{C}$ NMR $\left(100 \mathrm{MHz}, \mathrm{CDCl}_{3}\right): \delta=164.0,150.8$, 149.7, 148.5, 141.4, 139.7, 135.6, 130.5, 129.7, 128.1, 127.4, 123.6, 123.3, 119.4, 35.2, 31.4, 28.5, 22.0, 13.7. HRMS: [M-BF $]^{+}$ calculated for $\mathrm{C}_{19} \mathrm{H}_{21} \mathrm{~N}_{2} 277.1699$, found 277.1697. 
3ac, yellow solid (60.0 mg, 81\% yield). ${ }^{1} \mathrm{H}$ NMR (400 MHz, $\left.\mathrm{CDCl}_{3}\right): \delta=9.30(\mathrm{~d}, J=8.5 \mathrm{~Hz}, 1 \mathrm{H}), 8.68(\mathrm{~d}, J=4.6 \mathrm{~Hz}, 1 \mathrm{H}), 8.40$ $(\mathrm{d}, J=8.1 \mathrm{~Hz}, 1 \mathrm{H}), 8.12(\mathrm{~d}, J=8.5 \mathrm{~Hz}, 1 \mathrm{H}), 7.98(\mathrm{t}, J=7.9 \mathrm{~Hz}, 1 \mathrm{H})$, 7.94-7.87 (m, 2H), $7.76(\mathrm{~d}, J=7.9 \mathrm{~Hz}, 1 \mathrm{H}), 7.55(\mathrm{dd}, J=7.4,5.0$ $\mathrm{Hz}, 1 \mathrm{H}), 7.49$ (d, $J=7.3 \mathrm{~Hz}, 2 \mathrm{H}), 7.38$ (d, $J=7.3 \mathrm{~Hz}, 1 \mathrm{H})$, 7.35-7.30 (m, 3H). ${ }^{13} \mathrm{C}$ NMR (100 MHz, $\left.\mathrm{CDCl}_{3}\right): \delta=159.4,150.9$, 149.7, 149.1, 140.8, 139.9, 136.1, 132.4, 131.3, 130.8, 130.1, $130.0,129.1,128.9,126.7,125.2,124.5,119.9$. HRMS: [M-BF $]^{+}$ calculated for $\mathrm{C}_{20} \mathrm{H}_{15} \mathrm{~N}_{2} 283.1230$, found 283.1230 .

3ad, brown solid (39.0 mg, 58\% yield). ${ }^{1} \mathrm{H}$ NMR (400 MHz, $\mathrm{CDCl}_{3}$ ): $\delta=8.81(\mathrm{~d}, J=4.3 \mathrm{~Hz}, 1 \mathrm{H}), 8.38(\mathrm{dd}, J=6.5,3.3 \mathrm{~Hz}, 1 \mathrm{H}$ ), $8.28(\mathrm{t}, J=7.4 \mathrm{~Hz}, 1 \mathrm{H}), 8.00(\mathrm{~s}, 1 \mathrm{H}), 7.94-7.86(\mathrm{~m}, 3 \mathrm{H}), 7.82(\mathrm{dd}$, $J=7.5,5.0 \mathrm{~Hz}, 1 \mathrm{H}), 7.06(\mathrm{dd}, J=6.5,3.3 \mathrm{~Hz}, 1 \mathrm{H}), 3.07$ (s, 3H), $2.98(\mathrm{dq}, J=15.2,7.6 \mathrm{~Hz}, 1 \mathrm{H}), 2.73(\mathrm{dq}, J=15.2,7.5 \mathrm{~Hz}, 1 \mathrm{H})$, $1.37(\mathrm{t}, J=7.5 \mathrm{~Hz}, 3 \mathrm{H}) .{ }^{13} \mathrm{C}$ NMR $\left(100 \mathrm{MHz}, \mathrm{CDCl}_{3}\right): \delta=163.4$, $160.0,150.9,149.7,141.4,139.1,135.0,129.5,127.7,127.3$, $126.4,123.8,123.5,119.9,28.5,20.5,12.7$. HRMS: $\left[{\left.\mathrm{M}-B F_{4}\right]^{+}}^{+}\right.$ calculated for $\mathrm{C}_{17} \mathrm{H}_{17} \mathrm{~N}_{2} 249.1386$, found 249.1387 .

3ae, white solid (36.9 mg, 51\% yield). ${ }^{1} \mathrm{H}$ NMR (400 MHz, $\left.\mathrm{CDCl}_{3}\right): \delta=8.81(\mathrm{~d}, J=4.6 \mathrm{~Hz}, 1 \mathrm{H}), 8.38(\mathrm{dd}, J=6.3,3.4 \mathrm{~Hz}, 1 \mathrm{H})$, 8.30 (td, $J=7.8,1.6 \mathrm{~Hz}, 1 \mathrm{H}), 8.00-7.94(\mathrm{~m}, 2 \mathrm{H}), 7.92-7.87(\mathrm{~m}$, $2 \mathrm{H}$ ), 7.83 (dd, $J=7.4,5.0 \mathrm{~Hz}, 1 \mathrm{H}$ ), 7.06 (dd, $J=6.7,3.2 \mathrm{~Hz}, 1 \mathrm{H}$ ), 3.07 (s, 3H), 3.04-2.95 (m, 1H), 2.72-2.63 (m, 1H), 1.79-1.71 (m, 2H), $1.33-1.25(\mathrm{~m}, 2 \mathrm{H}), 0.80(\mathrm{t}, J=7.3 \mathrm{~Hz}, 3 \mathrm{H}) .{ }^{13} \mathrm{C}$ NMR (100 MHz, $\left.\mathrm{CDCl}_{3}\right): \delta=162.6,159.5,150.7,149.7,141.3,139.1$, $134.9,129.4,127.7,127.3,126.31,124.4,123.6,120.0,34.7$, 30.8, 22.5, 20.4, 13.4. HRMS: $\left[\mathrm{M}-\mathrm{BF}_{4}\right]^{+}$calculated for $\mathrm{C}_{19} \mathrm{H}_{21} \mathrm{~N}_{2}$ 277.1699, found 277.1700.

3ba, yellow solid (57.0 mg, 85\% yield). ${ }^{1} \mathrm{H}$ NMR (400 MHz, $\left.\mathrm{CDCl}_{3}\right): \delta=9.10(\mathrm{~d}, J=8.7 \mathrm{~Hz}, 1 \mathrm{H}), 8.82(\mathrm{~d}, J=4.2 \mathrm{~Hz}, 1 \mathrm{H})$, 8.36-8.28 (m, 1H), 8.13-8.06 (m, 2H), $7.92(\mathrm{~d}, J=7.9 \mathrm{~Hz}, 1 \mathrm{H})$, 7.85 (dd, $J=7.4,5.0 \mathrm{~Hz}, 1 \mathrm{H}), 7.74$ (d, $J=9.0 \mathrm{~Hz}, 1 \mathrm{H}), 6.99$ (d, $J=$ $9.0 \mathrm{~Hz}, 1 \mathrm{H}), 3.06-2.96(\mathrm{~m}, 1 \mathrm{H}), 2.80-2.71(\mathrm{~m}, 1 \mathrm{H}), 2.58(\mathrm{~s}, 3 \mathrm{H})$, $1.37(\mathrm{t}, J=7.5 \mathrm{~Hz}, 3 \mathrm{H}) .{ }^{13} \mathrm{C} \mathrm{NMR}\left(100 \mathrm{MHz}, \mathrm{CDCl}_{3}\right): \delta=163.5$, $150.9,149.6,147.9,141.4,140.6,138.2,137.7,129.1,128.3$, $127.4,123.1,122.8,119.1,28.5,21.3,12.6$. HRMS: [M-BF 4$]^{+}$ calculated for $\mathrm{C}_{17} \mathrm{H}_{17} \mathrm{~N}_{2} 249.1386$, found 249.1387 .

3ca, yellow solid (45.0 mg, 61\% yield). ${ }^{1} \mathrm{H}$ NMR (400 MHz, $\left.\mathrm{CDCl}_{3}\right): \delta=9.23(\mathrm{~d}, J=8.7 \mathrm{~Hz}, 1 \mathrm{H}), 8.82(\mathrm{~d}, J=3.9 \mathrm{~Hz}, 1 \mathrm{H}), 8.34$ $(\mathrm{t}, J=7.2 \mathrm{~Hz}, 1 \mathrm{H}), 8.12-8.04(\mathrm{~m}, 2 \mathrm{H}), 7.83(\mathrm{dd}, J=7.4,4.9 \mathrm{~Hz}$, 1H), 7.72 (d, $J=2.4 \mathrm{~Hz}, 1 \mathrm{H}$ ), 7.47 (dd, $J=9.6,2.5 \mathrm{~Hz}, 1 \mathrm{H}$ ), 6.95 (d, $J=9.6 \mathrm{~Hz}, 1 \mathrm{H}), 4.29-4.19(\mathrm{~m}, 2 \mathrm{H}), 3.09-2.96(\mathrm{~m}, 1 \mathrm{H})$, 2.78-2.71 (m, 1H), $1.48(\mathrm{t}, J=6.9 \mathrm{~Hz}, 3 \mathrm{H}), 1.37(\mathrm{t}, J=7.5 \mathrm{~Hz}$, 3H). ${ }^{13} \mathrm{C}$ NMR (100 MHz, $\left.\mathrm{CDCl}_{3}\right): \delta=161.1,159.1,150.9,149.9$, 147.4, 141.5, 135.1, 130.2, 128.3, 127.3, 123.4, 123.1, 120.6,

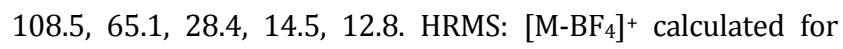
$\mathrm{C}_{18} \mathrm{H}_{19} \mathrm{~N}_{2} \mathrm{O} 279.1492$, found 279.1494 .

3da, brown solid (53.0 mg, 74\% yield). ${ }^{1} \mathrm{H}$ NMR (400 MHz, $\mathrm{CDCl}_{3}$ ): $\delta=9.05(\mathrm{~d}, J=8.7 \mathrm{~Hz}, 1 \mathrm{H}), 8.73(\mathrm{~d}, J=3.8 \mathrm{~Hz}, 1 \mathrm{H}), 8.20$ (d, $J=9.1 \mathrm{~Hz}, 2 \mathrm{H}), 8.07(\mathrm{~d}, J=8.8 \mathrm{~Hz}, 1 \mathrm{H}), 7.95(\mathrm{~d}, J=7.9 \mathrm{~Hz}$, 1H), 7.78-7.68 (m, 2H), 6.99 (d, $J=9.3 \mathrm{~Hz}, 1 \mathrm{H}), 2.94$ (dd, $J=$ 15.9, $7.7 \mathrm{~Hz}, 1 \mathrm{H}$ ), 2.69 (dd, $J=15.7,7.7 \mathrm{~Hz}, 1 \mathrm{H}$ ), 1.30 (t, $J=7.5$ $\mathrm{Hz}, 3 \mathrm{H}) .{ }^{13} \mathrm{C}$ NMR $\left(100 \mathrm{MHz}, \mathrm{CDCl}_{3}\right): \delta=165.4,151.0,149.5$, 147.6, 141.5, 138.2, 135.8, 128.9, 127.6, 124.2, 123.3, 121.3, 28.9, 12.5. HRMS: $\left[\mathrm{M}-\mathrm{BF}_{4}\right]^{+}$calculated for $\mathrm{C}_{16} \mathrm{H}_{14} \mathrm{ClN}_{2} 269.0840$, found 269.0841 .

3ea, green solid (66.0 mg, 83\% yield). ${ }^{1} \mathrm{H}$ NMR (400 MHz,
$\left.\mathrm{CDCl}_{3}\right): \delta=9.23(\mathrm{~d}, J=8.7 \mathrm{~Hz}, 1 \mathrm{H}), 8.82(\mathrm{~d}, J=3.9 \mathrm{~Hz}, 1 \mathrm{H}), 8.34$ $(\mathrm{t}, J=7.2 \mathrm{~Hz}, 1 \mathrm{H}), 8.12-8.04(\mathrm{~m}, 2 \mathrm{H}), 7.83(\mathrm{dd}, J=7.4,4.9 \mathrm{~Hz}$, 1H), $7.72(\mathrm{~d}, J=2.4 \mathrm{~Hz}, 1 \mathrm{H}$ ), 7.47 (dd, $J=9.6,2.5 \mathrm{~Hz}, 1 \mathrm{H}$ ), 6.95 (d, $J=9.6 \mathrm{~Hz}, 1 \mathrm{H}), 4.29-4.19(\mathrm{~m}, 2 \mathrm{H}), 3.09-2.96(\mathrm{~m}, 1 \mathrm{H})$, 2.78-2.71 (m, 1H), $1.48(\mathrm{t}, J=6.9 \mathrm{~Hz}, 3 \mathrm{H}), 1.37(\mathrm{t}, J=7.5 \mathrm{~Hz}$, 3H). ${ }^{13} \mathrm{C}$ NMR (100 MHz, $\left.\mathrm{CDCl}_{3}\right): \delta=161.1,159.1,150.9,149.9$, $147.4,141.5,135.1,130.2,128.3,127.3,123.4,123.1,120.6$, 108.5, 65.1, 28.4, 14.5, 12.8. HRMS: $\left[\mathrm{M}-\mathrm{BF}_{4}\right]^{+}$calculated for $\mathrm{C}_{16} \mathrm{H}_{14} \mathrm{BrN}_{2}$ 313.0335, found 313.0337.

3fa, yellow solid (55.5 mg, 83\% yield). ${ }^{1} \mathrm{H}$ NMR (400 MHz, $\left.\mathrm{CDCl}_{3}\right): \delta=9.15(\mathrm{~d}, J=8.6 \mathrm{~Hz}, 1 \mathrm{H}), 8.84(\mathrm{~d}, J=4.6 \mathrm{~Hz}, 1 \mathrm{H})$, $8.38-8.31(\mathrm{~m}, 1 \mathrm{H}), 8.23(\mathrm{~d}, J=8.4 \mathrm{~Hz}, 1 \mathrm{H}), 8.06(\mathrm{~d}, J=8.6 \mathrm{~Hz}$, 1H), 7.95 (d, $J=7.9 \mathrm{~Hz}, 1 \mathrm{H}), 7.87(\mathrm{dd}, J=7.5,4.9 \mathrm{~Hz}, 1 \mathrm{H}), 7.69$ (d, $J=8.3 \mathrm{~Hz}, 1 \mathrm{H}), 6.80(\mathrm{~s}, 1 \mathrm{H}), 3.04-2.95(\mathrm{~m}, 1 \mathrm{H}), 2.81-2.72(\mathrm{~m}$, $1 \mathrm{H}), 2.50(\mathrm{~s}, 3 \mathrm{H}), 1.37$ (t, $J=7.5 \mathrm{~Hz}, 3 \mathrm{H}) .{ }^{13} \mathrm{C}$ NMR $(100 \mathrm{MHz}$, $\left.\mathrm{CDCl}_{3}\right): \delta=164.0,151.0,149.6,148.3,148.1,141.5,140.0$, 131.8, 130.2, 127.4, 126.5, 123.3, 121.9, 118.3, 28.6, 22.9, 12.6. HRMS: $\left[\mathrm{M}-\mathrm{BF}_{4}\right]^{+}$calculated for $\mathrm{C}_{17} \mathrm{H}_{17} \mathrm{~N}_{2}$ 249.1386, found 249.1385.

3ga, brown solid (68.0 mg, 85\% yield). ${ }^{1} \mathrm{H}$ NMR (400 MHz, $\mathrm{CD}_{2} \mathrm{Cl}_{2}$ ): $\delta=9.12(\mathrm{~d}, J=8.6 \mathrm{~Hz}, 1 \mathrm{H}), 8.78(\mathrm{~d}, J=4.1 \mathrm{~Hz}, 1 \mathrm{H}), 8.33$ (d, $J=8.5 \mathrm{~Hz}, 1 \mathrm{H}), 8.23(\mathrm{t}, J=7.7 \mathrm{~Hz}, 1 \mathrm{H}), 8.07-7.97(\mathrm{~m}, 2 \mathrm{H})$, 7.81-7.71 (m, 2H), 7.43-7.31 (m, 5H), 7.09 (s, 1H), 2.88 (dq, $J=$ 15.0, 7.6 Hz, 1H), $2.73(\mathrm{dq}, J=15.2,7.5 \mathrm{~Hz}, 1 \mathrm{H}), 1.30(\mathrm{t}, J=7.5$ $\mathrm{Hz}, 3 \mathrm{H}) .{ }^{13} \mathrm{C}$ NMR $\left(100 \mathrm{MHz}, \mathrm{CD}_{2} \mathrm{Cl}_{2}\right): \delta=164.8,151.4,149.6$, $148.7,148.3,141.4,140.3,138.0,130.9,129.8,129.5,129.4$, 127.8, 127.5, 127.4, 122.9, 122.5, 116.6, 28.8, 12.6. HRMS: [M-BF $]^{+}$calculated for $\mathrm{C}_{23} \mathrm{H}_{19} \mathrm{~N}_{2} 311.1543$, found 311.1545.

3ha, yellow solid (62.0 mg, 89\% yield). ${ }^{1} \mathrm{H}$ NMR (400 MHz, $\left.\mathrm{CDCl}_{3}\right): \delta=9.07(\mathrm{~d}, J=8.6 \mathrm{~Hz}, 1 \mathrm{H}), 8.84(\mathrm{~d}, J=4.5 \mathrm{~Hz}, 1 \mathrm{H}), 8.35$ $(\mathrm{t}, J=7.7 \mathrm{~Hz}, 1 \mathrm{H}), 8.06(\mathrm{~s}, 1 \mathrm{H}), 8.03(\mathrm{~d}, J=8.6 \mathrm{~Hz}, 1 \mathrm{H}), 7.93(\mathrm{~d}, J$ $=7.9 \mathrm{~Hz}, 1 \mathrm{H}), 7.86(\mathrm{dd}, J=7.4,5.1 \mathrm{~Hz}, 1 \mathrm{H}), 6.80(\mathrm{~s}, 1 \mathrm{H})$, 3.01-2.91 (m, 1H), 2.78-2.70 (m, 1H), 2.49 (s, 3H), $2.40(\mathrm{~s}, 3 \mathrm{H})$, $1.36(\mathrm{t}, J=7.5 \mathrm{~Hz}, 3 \mathrm{H}) .{ }^{13} \mathrm{C}$ NMR $\left(100 \mathrm{MHz}, \mathrm{CDCl}_{3}\right): \delta=162.7$, $151.0,149.7,148.0,147.4,141.5,140.5,138.7,129.4,127.4$, 127.0, 123.2, 121.8, 118.7, 28.4, 21.5, 19.8, 12.6. HRMS: $\left[\mathrm{M}-\mathrm{BF}_{4}\right]^{+}$calculated for $\mathrm{C}_{18} \mathrm{H}_{19} \mathrm{~N}_{2} 311.1543$, found 311.1545 .

3ia, brown solid (60.0 mg, 83\% yield). ${ }^{1} \mathrm{H}$ NMR (400 MHz, $\left.\mathrm{CDCl}_{3}\right): \delta=9.11(\mathrm{~d}, J=8.6 \mathrm{~Hz}, 1 \mathrm{H}), 8.84(\mathrm{~d}, J=4.3 \mathrm{~Hz}, 1 \mathrm{H}), 8.36$ $(\mathrm{t}, J=7.7 \mathrm{~Hz}, 1 \mathrm{H}), 8.11(\mathrm{~s}, 1 \mathrm{H}), 8.04(\mathrm{~d}, J=8.6 \mathrm{~Hz}, 1 \mathrm{H}), 7.95-7.84$ $(\mathrm{m}, 2 \mathrm{H}), 6.86(\mathrm{~s}, 1 \mathrm{H}), 3.14(\mathrm{t}, J=7.4 \mathrm{~Hz}, 2 \mathrm{H}), 3.05(\mathrm{t}, J=7.4 \mathrm{~Hz}$, $2 \mathrm{H}), 2.96(\mathrm{dq}, J=15.9,7.7 \mathrm{~Hz}, 1 \mathrm{H}), 2.75(\mathrm{dq}, J=15.9,7.7 \mathrm{~Hz}$, $1 \mathrm{H}), 2.22-2.14(\mathrm{~m}, 2 \mathrm{H}), 1.37(\mathrm{t}, J=7.5 \mathrm{~Hz}, 3 \mathrm{H}) .{ }^{13} \mathrm{C}$ NMR $(100$ $\mathrm{MHz}, \mathrm{CDCl}_{3}$ ): $\delta=162.3,155.9,151.0,149.8,148.0,147.8,141.5$, 139.6, 127.8, 127.3, 124.4, 123.1, 121.4, 114.0, 33.8, 32.2, 28.3, 25.7, 12.6. HRMS: [M-BF $]^{+}$calculated for $\mathrm{C}_{18} \mathrm{H}_{19} \mathrm{~N}_{2} 275.1543$, found 275.1544 .

4af, Brown solid (41.2 mg, 92\% yield). ${ }^{1} \mathrm{H}$ NMR (400 MHz, $\left.\mathrm{CDCl}_{3}\right): \delta=8.66(\mathrm{~d}, J=3.4 \mathrm{~Hz}, 1 \mathrm{H}), 7.92-7.85(\mathrm{~m}, 1 \mathrm{H}), 7.41-7.32$ (m, 2H), 7.21 (d, $J=6.7 \mathrm{~Hz}, 1 \mathrm{H}), 7.08-6.96(\mathrm{~m}, 2 \mathrm{H}), 6.31-6.22$ (m, 1H), 3.12-3.03 (m, 2H), 2.88-2.79 (m, 2H). ${ }^{13} \mathrm{C}$ NMR $(100$ $\mathrm{MHz}, \mathrm{CDCl}_{3}$ ): $\delta=170.5,152.0,150.2,140.5,138.8,128.0,127.2$, $125.9,124.7,123.4,123.3,117.0,32.3,25.6$. HRMS: $[\mathrm{M}+\mathrm{H}]^{+}$ calculated for $\mathrm{C}_{14} \mathrm{H}_{13} \mathrm{~N}_{2} \mathrm{O} 225.1028$, found 225.1030 .

4bf, brown oil (40.0 mg, 84\% yield). ${ }^{1} \mathrm{H}$ NMR (400 MHz, $\left.\mathrm{CDCl}_{3}\right): \delta=8.65(\mathrm{~s}, 1 \mathrm{H}), 7.92-7.84(\mathrm{~m}, 1 \mathrm{H}), 7.44-7.30(\mathrm{~m}, 2 \mathrm{H})$, $7.03(\mathrm{~s}, 1 \mathrm{H}), 6.85(\mathrm{dd}, J=8.2,1.3 \mathrm{~Hz}, 1 \mathrm{H}), 6.17(\mathrm{~d}, J=8.2 \mathrm{~Hz}, 1 \mathrm{H})$, 
3.07-3.02 (m, 2H), 2.84-2.77 (m, 2H), $2.27(\mathrm{~s}, 3 \mathrm{H}) .{ }^{13} \mathrm{C}$ NMR (100 MHz, $\left.\mathrm{CDCl}_{3}\right): \delta=170.4,152.1,150.1,138.7,138.2,133.1$, 128.7, 127.6, 125.9, 124.6, 123.4, 117.0, 32.5, 25.7, 20.7. HRMS: $[\mathrm{M}+\mathrm{H}]^{+}$calculated for $\mathrm{C}_{15} \mathrm{H}_{15} \mathrm{~N}_{2} \mathrm{O} 239.1184$, found 239.1181 .

4cf, yellow solid (40.0 mg, 92\% yield). ${ }^{1} \mathrm{H}$ NMR (400 MHz, $\left.\mathrm{CDCl}_{3}\right): \delta=8.65(\mathrm{~s}, 1 \mathrm{H}), 7.87(\mathrm{t}, J=7.3 \mathrm{~Hz}, 1 \mathrm{H}), 7.42-7.31(\mathrm{~m}$, $2 \mathrm{H}), 7.22(\mathrm{~s}, 1 \mathrm{H}), 7.07(\mathrm{~d}, J=8.3 \mathrm{~Hz}, 1 \mathrm{H}), 6.21(\mathrm{~d}, J=8.5 \mathrm{~Hz}, 1 \mathrm{H})$, $3.08(\mathrm{t}, J=7.1 \mathrm{~Hz}, 2 \mathrm{H}), 2.83(\mathrm{t}, J=7.1 \mathrm{~Hz}, 2 \mathrm{H}), 1.28(\mathrm{~s}, 9 \mathrm{H}) .{ }^{13} \mathrm{C}$ NMR (100 MHz, $\left.\mathrm{CDCl}_{3}\right): \delta=170.4,152.1,150.1,146.4,138.6$, 138.1, 125.4, 125.0, 124.6, 124.0, 123.3, 116.6, 34.3, 32.5, 31.4, 25.9. HRMS: $[\mathrm{M}+\mathrm{H}]+$ calculated for $\mathrm{C}_{18} \mathrm{H}_{21} \mathrm{~N}_{2} \mathrm{O}$ 281.1654, found 281.1656 .

4df, yellow solid (41.8 mg, 78\% yield). ${ }^{1} \mathrm{H}$ NMR (400 MHz, $\left.\mathrm{CDCl}_{3}\right): \delta=8.64(\mathrm{~d}, J=3.6 \mathrm{~Hz}, 1 \mathrm{H}), 7.93-7.82(\mathrm{~m}, 1 \mathrm{H}), 7.44-7.30$ $(\mathrm{m}, 2 \mathrm{H}), 6.77(\mathrm{~d}, J=2.6 \mathrm{~Hz}, 1 \mathrm{H}), 6.58(\mathrm{dd}, J=8.8,2.7 \mathrm{~Hz}, 1 \mathrm{H})$, $6.21(\mathrm{~d}, J=8.8 \mathrm{~Hz}, 1 \mathrm{H}), 3.97(\mathrm{q}, J=7.0 \mathrm{~Hz}, 2 \mathrm{H}), 3.16-2.98(\mathrm{~m}$, $2 \mathrm{H}), 2.85-2.75(\mathrm{~m}, 2 \mathrm{H}), 1.38(\mathrm{t}, J=7.0 \mathrm{~Hz}, 3 \mathrm{H}) .{ }^{13} \mathrm{C}$ NMR $(100$ $\left.\mathrm{MHz}, \mathrm{CDCl}_{3}\right): \delta=170.1,155.1,152.1,150.1,138.7,134.1,127.5$, 124.6, 123.3, 118.1, 114.3, 112.8, 63.8, 32.4, 26.0, 14.9. HRMS: $[\mathrm{M}+\mathrm{H}]^{+}$calculated for $\mathrm{C}_{16} \mathrm{H}_{17} \mathrm{~N}_{2} \mathrm{O}_{2} 269.1290$, found 269.1291 .

4ef, Yellow solid (48.5 mg, 94\% yield). ${ }^{1} \mathrm{H}$ NMR (400 MHz, $\left.\mathrm{CDCl}_{3}\right): \delta=8.64(\mathrm{~d}, J=4.7 \mathrm{~Hz}, 1 \mathrm{H}), 7.93-7.87(\mathrm{~m}, 1 \mathrm{H}), 7.40-7.35$ (m, $2 \mathrm{H}), 7.21(\mathrm{~d}, J=2.3 \mathrm{~Hz}, 1 \mathrm{H}), 7.01(\mathrm{dd}, J=8.7,2.4 \mathrm{~Hz}, 1 \mathrm{H})$, $6.23(\mathrm{~d}, J=8.7 \mathrm{~Hz}, 1 \mathrm{H}), 3.09-3.04(\mathrm{~m}, 2 \mathrm{H}), 2.85-2.79(\mathrm{~m}, 2 \mathrm{H})$. ${ }^{13} \mathrm{C}$ NMR $\left(100 \mathrm{MHz}, \mathrm{CDCl}_{3}\right): \delta=170.0,151.6,150.2,139.2$, 138.9, 128.6, 127.9, 127.7, 127.1, 124.6, 123.6, 118.3, 32.0, 25.5. HRMS: $[\mathrm{M}+\mathrm{H}]^{+}$calculated for $\mathrm{C}_{14} \mathrm{H}_{12} \mathrm{ClN}_{2} \mathrm{O}$ 259.0638, found 259.0636 .

4ff, yellow solid (48.3 mg, 80\% yield). ${ }^{1} \mathrm{H}$ NMR (400 MHz, $\left.\mathrm{CDCl}_{3}\right): \delta=8.64(\mathrm{~d}, J=4.3 \mathrm{~Hz}, 1 \mathrm{H}), 7.93-7.86(\mathrm{~m}, 1 \mathrm{H}), 7.37$ (dd, $J$ $=7.6,4.6 \mathrm{~Hz}, 3 \mathrm{H}), 7.16(\mathrm{dd}, J=8.7,2.0 \mathrm{~Hz}, 1 \mathrm{H}), 6.17(\mathrm{~d}, J=8.7$ $\mathrm{Hz}, 1 \mathrm{H}), 3.09-3.04(\mathrm{~m}, 2 \mathrm{H}), 2.84-2.79(\mathrm{~m}, 2 \mathrm{H}) .{ }^{13} \mathrm{C}$ NMR (100 $\left.\mathrm{MHz}, \mathrm{CDCl}_{3}\right): \delta=169.9,151.6,150.3,139.7,138.9,130.8,130.1$, 128.1, 124.5, 123.6, 118.7, 116.2, 32.0, 25.4. HRMS: $[\mathrm{M}+\mathrm{H}]^{+}$ calculated for $\mathrm{C}_{14} \mathrm{H}_{12} \mathrm{BrN}_{2} \mathrm{O} 303.0133$, found 303.0133.

4gf, yellow solid (54.0 mg, 90\% yield). ${ }^{1} \mathrm{H}$ NMR (400 MHz, $\left.\mathrm{CDCl}_{3}\right): \delta=8.67(\mathrm{~s}, 1 \mathrm{H}), 7.89(\mathrm{t}, J=7.6 \mathrm{~Hz}, 1 \mathrm{H}), 7.48-7.32(\mathrm{~m}$, $6 \mathrm{H}), 7.27$ (d, $J=7.5 \mathrm{~Hz}, 2 \mathrm{H}$ ), 7.21 (dd, $J=7.7,1.3 \mathrm{~Hz}, 1 \mathrm{H}$ ), 6.46 (d, $J=0.9 \mathrm{~Hz}, 1 \mathrm{H}), 3.18-3.04(\mathrm{~m}, 2 \mathrm{H}), 2.91-2.81(\mathrm{~m}, 2 \mathrm{H}) .{ }^{13} \mathrm{C}$ NMR $\left(100 \mathrm{MHz}, \mathrm{CDCl}_{3}\right): \delta=170.5,151.9,150.2,140.9,140.7$, 140.5, 138.8, 128.73, 128.4, 127.4, 127.1, 125.0, 124.7, 123.6, 122.4, 115.9, 32.4, 25.4. HRMS: $[\mathrm{M}+\mathrm{H}]^{+}$calculated for $\mathrm{C}_{20} \mathrm{H}_{17} \mathrm{~N}_{2} \mathrm{O} 301.1341$, found 301.1342 .

4hf, white solid (44.4 mg, 86\% yield). ${ }^{1} \mathrm{H}$ NMR (400 MHz, $\left.\mathrm{CDCl}_{3}\right): \delta=8.67(\mathrm{~s}, 1 \mathrm{H}), 7.95-7.87(\mathrm{~m}, 1 \mathrm{H}), 7.44-7.34(\mathrm{~m}, 2 \mathrm{H})$, $7.14(\mathrm{~d}, J=8.0 \mathrm{~Hz}, 1 \mathrm{H}), 6.97(\mathrm{dd}, J=8.0,1.9 \mathrm{~Hz}, 1 \mathrm{H}), 6.27(\mathrm{~d}, J=$ $1.9 \mathrm{~Hz}, 1 \mathrm{H}), 3.10-3.01(\mathrm{~m}, 2 \mathrm{H}), 2.85-2.78(\mathrm{~m}, 2 \mathrm{H}) .{ }^{13} \mathrm{C}$ NMR $\left(100 \mathrm{MHz}, \mathrm{CDCl}_{3}\right): \delta=170.1,151.4,150.4,141.5,139.0,132.7$, 129.1, 124.6, 124.3, 123.8, 123.3, 117.2, 32.1, 25.2. HRMS: $[\mathrm{M}+\mathrm{H}]^{+}$calculated for $\mathrm{C}_{14} \mathrm{H}_{12} \mathrm{ClN}_{2} \mathrm{O} 259.0638$, found 259.0638 .

4if, white solid (45.2 mg, 95\% yield). ${ }^{1} \mathrm{H}$ NMR (400 MHz, $\left.\mathrm{CDCl}_{3}\right): \delta=8.67(\mathrm{~d}, J=3.3 \mathrm{~Hz}, 1 \mathrm{H}), 7.95-7.85(\mathrm{~m}, 1 \mathrm{H}), 7.44-7.33$ (m, 2H), $7.09(\mathrm{~d}, J=7.6 \mathrm{~Hz}, 1 \mathrm{H}), 6.81(\mathrm{~d}, J=7.5 \mathrm{~Hz}, 1 \mathrm{H}), 6.06(\mathrm{~s}$, 1H), 3.11-2.97 (m, 2H), 2.86-2.76 (m, 2H), $2.16(\mathrm{~s}, 3 \mathrm{H}) .{ }^{13} \mathrm{C}$ NMR (100 MHz, $\left.\mathrm{CDCl}_{3}\right): \delta=170.6,152.0,150.2,140.4,138.7$, 137.0, 127.8, 124.7, 124.1, 123.4, 122.9, 117.6, 32.5, 25.2, 21.3. HRMS: $[\mathrm{M}+\mathrm{H}]^{+}$calculated for $\mathrm{C}_{15} \mathrm{H}_{15} \mathrm{~N}_{2} \mathrm{O}$ 239.1184, found

\subsection{1.}

4jf, white solid (42.8 mg, 85\% yield). ${ }^{1} \mathrm{H}$ NMR (400 MHz, $\left.\mathrm{CDCl}_{3}\right): \delta=8.66(\mathrm{~s}, 1 \mathrm{H}), 7.89(\mathrm{t}, J=7.6 \mathrm{~Hz}, 1 \mathrm{H}), 7.44-7.31(\mathrm{~m}$, 2H), 6.97 (s, 1H), $6.04(\mathrm{~s}, 1 \mathrm{H}), 3.03-2.98(\mathrm{~m}, 2 \mathrm{H}), 2.82-2.76(\mathrm{~m}$, 2H), $2.18(\mathrm{~s}, 3 \mathrm{H}), 2.06(\mathrm{~s}, 3 \mathrm{H}) .{ }^{13} \mathrm{C}$ NMR $\left(100 \mathrm{MHz}, \mathrm{CDCl}_{3}\right): \delta=$ 170.5, 152.1, 150.1, 138.7, 138.3, 135.3, 131.7, 129.1, 124.7, 123.4, 123.2, 118.3, 32.7, 25.2, 19.7, 19.0. HRMS: [M+H]+ calculated for $\mathrm{C}_{16} \mathrm{H}_{17} \mathrm{~N}_{2} \mathrm{O} 253.1341$, found 253.1341 .

4kf, white solid (46.5 mg, 88\% yield). ${ }^{1} \mathrm{H}$ NMR $(400 \mathrm{MHz}$, $\left.\mathrm{CDCl}_{3}\right): \delta=8.66(\mathrm{~s}, 1 \mathrm{H}), 7.89(\mathrm{t}, J=7.6 \mathrm{~Hz}, 1 \mathrm{H}), 7.46-7.31(\mathrm{~m}$, 2H), 7.07 (s, 1H), 6.13 (s, 1H), 3.08-3.01 (m, 2H), 2.86-2.77 (m, $4 \mathrm{H}), 2.71(\mathrm{t}, J=7.4 \mathrm{~Hz}, 2 \mathrm{H}), 2.01(\mathrm{t}, J=7.3 \mathrm{~Hz}, 2 \mathrm{H}) .{ }^{13} \mathrm{C} \mathrm{NMR}$ $\left(100 \mathrm{MHz}, \mathrm{CDCl}_{3}\right): \delta=170.7,152.3,150.1,143.3,139.4,139.0$, 138.7, 124.7, 124.1, 123.8, 123.3, 113.3, 32.9, 32.7, 32.3, 25.8, 25.7. HRMS: $[\mathrm{M}+\mathrm{H}]^{+}$calculated for $\mathrm{C}_{17} \mathrm{H}_{17} \mathrm{~N}_{2} \mathrm{O} 265.1341$, found 265.1342 .

4lf, brown solid (43.9 mg, 82\% yield). ${ }^{1} \mathrm{H}$ NMR (400 MHz, $\left.\mathrm{CDCl}_{3}\right): \delta=8.65(\mathrm{~s}, 1 \mathrm{H}), 7.92-7.85(\mathrm{~m}, 1 \mathrm{H}), 7.42-7.31(\mathrm{~m}, 2 \mathrm{H})$, $6.50(\mathrm{~d}, J=8.4 \mathrm{~Hz}, 1 \mathrm{H}), 5.97(\mathrm{~s}, 2 \mathrm{H}), 5.71(\mathrm{~d}, J=8.4 \mathrm{~Hz}, 1 \mathrm{H})$, 3.09-2.99 (m, 2H), 2.85-2.77 (m, 2H). ${ }^{13} \mathrm{C}$ NMR (100 MHz, $\left.\mathrm{CDCl}_{3}\right): \delta=169.7,152.2,150.2,144.8,143.6,138.8,135.9$, 124.6, 123.5, 109.7, 108.9, 106.2, 101.6, 31.7, 18.9. HRMS: $[\mathrm{M}+\mathrm{H}]^{+}$calculated for $\mathrm{C}_{15} \mathrm{H}_{13} \mathrm{~N}_{2} \mathrm{O}_{3} 269.0926$, found 269.0926 .

4mf, white solid (50.0 mg, 85\% yield). ${ }^{1} \mathrm{H}$ NMR $(400 \mathrm{MHz}$, $\mathrm{CDCl}_{3}$ ): $\delta=8.66(\mathrm{~d}, J=3.6 \mathrm{~Hz}, 1 \mathrm{H}), 7.96-7.87(\mathrm{~m}, 1 \mathrm{H}), 7.43-7.35$ (m, 2H), 7.30 (s, 1H), 6.38 (s, 1H), 3.10-3.00 (m, 2H), 2.86-2.79 (m, 2H). ${ }^{13} \mathrm{C}$ NMR (100 MHz, $\left.\mathrm{CDCl}_{3}\right): \delta=169.7,151.1,150.4$, 140.0, 139.1, 130.9, 129.4, 126.7, 126.0, 124.5, 124.0, 118.8, 31.8, 25.1. HRMS: $[\mathrm{M}+\mathrm{H}]^{+}$calculated for $\mathrm{C}_{14} \mathrm{H}_{11} \mathrm{Cl}_{2} \mathrm{~N}_{2} \mathrm{O}$ 293.0248, found 293.0246.

4ag, brown oil (24.5 mg, 46\% yield). ${ }^{1} \mathrm{H}$ NMR (400 MHz, $\mathrm{CDCl}_{3}$ ): $\delta=8.66(\mathrm{~d}, J=4.3 \mathrm{~Hz}, 1 \mathrm{H}), 7.92-7.83(\mathrm{~m}, 1 \mathrm{H}), 7.38-7.32$ (m, 2H), 7.22-7.15 (m, 1H), 7.08-6.98 (m, 2H), 6.31 (d, $J=7.7$ $\mathrm{Hz}, 1 \mathrm{H}), 3.04-2.92(\mathrm{~m}, 2 \mathrm{H}), 2.73(\mathrm{dd}, J=15.0,3.3 \mathrm{~Hz}, 1 \mathrm{H})$, 1.79-1.72 (m, 1H), 1.70-1.62 (m, 1H), 1.55-1.46 (m, 1H), 1.44-1.35 (m, 1H), $0.95(\mathrm{t}, J=7.3 \mathrm{~Hz}, 3 \mathrm{H}) .{ }^{13} \mathrm{C}$ NMR $(100 \mathrm{MHz}$, $\left.\mathrm{CDCl}_{3}\right): \delta=169.9,152.1,150.2,139.7,138.7,130.0,127.9$, 127.2, 124.6, 123.4, 123.4, 117.5, 37.5, 36.3, 36.2, 20.2, 14.1 . HRMS: $[\mathrm{M}+\mathrm{H}]^{+}$calculated for $\mathrm{C}_{17} \mathrm{H}_{19} \mathrm{~N}_{2} \mathrm{O}$ 267.1497, found 267.1500 .

6aa, yellow solid (42.3 mg, 83\% yield). ${ }^{1} \mathrm{H}$ NMR (400 MHz, $\left.\mathrm{CDCl}_{3}\right): \delta=8.39(\mathrm{~d}, J=4.7 \mathrm{~Hz}, 2 \mathrm{H}), 7.57(\mathrm{~d}, J=8.0 \mathrm{~Hz}, 1 \mathrm{H})$, 7.19-7.12 (m, 2H), $7.02(\mathrm{t}, J=7.3 \mathrm{~Hz}, 1 \mathrm{H}), 6.69(\mathrm{t}, J=4.7 \mathrm{~Hz}$, $1 \mathrm{H}), 6.39(\mathrm{t}, J=3.7 \mathrm{~Hz}, 1 \mathrm{H}), 3.75-3.63(\mathrm{~m}, 2 \mathrm{H}), 3.01-2.87(\mathrm{~m}$, 1H), 2.77-2.64 (m, 1H), 2.20-2.05 (m, 2H), $1.13(\mathrm{t}, J=7.0 \mathrm{~Hz}$,

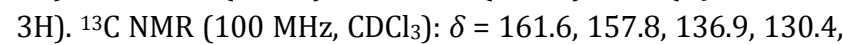
128.7, 125.5, 124.7, 123.8, 113.0, 82.0, 62.5, 29.1, 23.2, 15.3. HRMS: $[\mathrm{M}+\mathrm{H}]^{+}$calculated for $\mathrm{C}_{15} \mathrm{H}_{18} \mathrm{~N}_{3} \mathrm{O}$ 256.1450, found 256.1451.

6ba, yellow solid (42.5 mg, 79\% yield). ${ }^{1} \mathrm{H}$ NMR (400 MHz, $\left.\mathrm{CDCl}_{3}\right): \delta=8.38(\mathrm{~d}, J=4.7 \mathrm{~Hz}, 2 \mathrm{H}), 7.44(\mathrm{~d}, J=8.0 \mathrm{~Hz}, 1 \mathrm{H})$, $7.00-6.93(\mathrm{~m}, 2 \mathrm{H}), 6.66(\mathrm{t}, J=4.7 \mathrm{~Hz}, 1 \mathrm{H}), 6.39(\mathrm{t}, J=3.9 \mathrm{~Hz}$, $1 \mathrm{H}), 3.73-3.62(\mathrm{~m}, 2 \mathrm{H}), 2.96-2.83(\mathrm{~m}, 1 \mathrm{H}), 2.72-2.61(\mathrm{~m}, 1 \mathrm{H})$, 2.29 (s, 3H), 2.21-2.03 (m, 2H), $1.14(\mathrm{t}, J=7.0 \mathrm{~Hz}, 3 \mathrm{H}) .{ }^{13} \mathrm{C}$ NMR $\left(100 \mathrm{MHz}, \mathrm{CDCl}_{3}\right): \delta=161.7,157.8,134.3,133.3,130.4,129.1$, 126.3, 124.7, 112.7, 82.1, 62.5, 29.3, 23.3, 21.1, 15.3. HRMS: $[\mathrm{M}+\mathrm{H}]^{+}$calculated for $\mathrm{C}_{16} \mathrm{H}_{20} \mathrm{~N}_{3} \mathrm{O} 270.1606$, found 270.1609 . 
6ca, yellow solid (51.8 mg, 91\% yield). ${ }^{1} \mathrm{H}$ NMR (400 MHz, $\left.\mathrm{CDCl}_{3}\right): \delta=8.36(\mathrm{~d}, J=4.7 \mathrm{~Hz}, 2 \mathrm{H}), 7.48(\mathrm{~d}, J=8.8 \mathrm{~Hz}, 1 \mathrm{H}), 6.75$ $(\mathrm{d}, J=8.9 \mathrm{~Hz}, 1 \mathrm{H}), 6.69(\mathrm{~s}, 1 \mathrm{H}), 6.64(\mathrm{t}, J=4.7 \mathrm{~Hz}, 1 \mathrm{H}), 6.42(\mathrm{t}, J=$ $3.8 \mathrm{~Hz}, 1 \mathrm{H}), 3.78(\mathrm{~s}, 3 \mathrm{H}), 3.71-3.62(\mathrm{~m}, 2 \mathrm{H}), 2.93-2.83(\mathrm{~m}, 1 \mathrm{H})$, 2.73-2.62 (m, 1H), 2.24-2.15 (m, 1H), 2.11-2.01 (m, 1H), 1.13 $(\mathrm{t}, J=7.0 \mathrm{~Hz}, 3 \mathrm{H}) .{ }^{13} \mathrm{C} \mathrm{NMR}\left(100 \mathrm{MHz}, \mathrm{CDCl}_{3}\right): \delta=161.5,157.7$, $155.9,132.2,130.0,126.2,113.0,112.5,111.7,81.9,62.4,55.4$, 29.3, 23.7, 15.3. HRMS: $[\mathrm{M}+\mathrm{H}]^{+}$calculated for $\mathrm{C}_{16} \mathrm{H}_{20} \mathrm{~N}_{3} \mathrm{O}_{2}$ 286.1556, found 286.1558 .

6da, yellow solid (54.6 mg, 85\% yield). ${ }^{1} \mathrm{H}$ NMR (400 MHz, $\left.\mathrm{CDCl}_{3}\right): \delta=8.45(\mathrm{~d}, J=4.8 \mathrm{~Hz}, 2 \mathrm{H}), 7.70(\mathrm{~d}, J=8.5 \mathrm{~Hz}, 1 \mathrm{H})$, 7.44-7.34 (m, 2H), $6.78(\mathrm{t}, J=4.8 \mathrm{~Hz}, 1 \mathrm{H}), 6.37(\mathrm{t}, J=3.3 \mathrm{~Hz}$, $1 \mathrm{H}), 3.77-3.63(\mathrm{~m}, 2 \mathrm{H}), 3.13-3.01(\mathrm{~m}, 1 \mathrm{H}), 2.85-2.74(\mathrm{~m}, 1 \mathrm{H})$, 2.23-2.15 (m, 1H), 2.12-2.00 (m, 1H), $1.13(\mathrm{t}, J=7.0 \mathrm{~Hz}, 3 \mathrm{H})$. ${ }^{13} \mathrm{C}$ NMR (100 MHz, $\left.\mathrm{CDCl}_{3}\right): \delta=161.3,157.9,140.2,130.0,126.1$ $\left(\mathrm{q}, J_{\mathrm{C}-\mathrm{F}}=3.9 \mathrm{~Hz}\right), 124.5\left(\mathrm{~d}, J_{\mathrm{C}-\mathrm{F}}=270 \mathrm{~Hz}\right), 124.93\left(\mathrm{~d}, J_{\mathrm{C}-\mathrm{F}}=32.4\right.$ $\mathrm{Hz}), 124.06,122.5$ (q, JC-F $=3.7 \mathrm{~Hz}$ ), 114.0, 82.05, 62.9, 28.1, 23.0, 15.3. HRMS: $[\mathrm{M}+\mathrm{H}]^{+}$calculated for $\mathrm{C}_{16} \mathrm{H}_{17} \mathrm{~F}_{3} \mathrm{~N}_{3} \mathrm{O} 324.1324$, found 324.1327 .

6ea, yellow solid (40.3 mg, 75\% yield). ${ }^{1} \mathrm{H}$ NMR (400 MHz, $\left.\mathrm{CDCl}_{3}\right): \delta=8.40(\mathrm{~d}, J=4.7 \mathrm{~Hz}, 2 \mathrm{H}), 7.35(\mathrm{~s}, 1 \mathrm{H}), 7.03(\mathrm{~d}, J=7.7$ $\mathrm{Hz}, 1 \mathrm{H}), 6.85$ (d, $J=7.6 \mathrm{~Hz}, 1 \mathrm{H}), 6.69$ (t, $J=4.7 \mathrm{~Hz}, 1 \mathrm{H}), 6.36(\mathrm{t}, J$ $=3.9 \mathrm{~Hz}, 1 \mathrm{H}), 3.75-3.64(\mathrm{~m}, 2 \mathrm{H}), 2.93-2.83(\mathrm{~m}, 1 \mathrm{H}), 2.71-2.62$ (m, 1H), $2.31(\mathrm{~s}, 3 \mathrm{H}), 2.19-2.03(\mathrm{~m}, 2 \mathrm{H}), 1.14(\mathrm{t}, J=7.0 \mathrm{~Hz}, 3 \mathrm{H})$. ${ }^{13} \mathrm{C}$ NMR $\left(100 \mathrm{MHz}, \mathrm{CDCl}_{3}\right): \delta=161.7,157.8,136.7,134.9$, 128.5, 127.5, 125.0, 113.3, 112.9, 82.3, 62.6, 29.4, 22.9, 21.5, 15.3. HRMS: $[\mathrm{M}+\mathrm{H}]^{+}$calculated for $\mathrm{C}_{16} \mathrm{H}_{20} \mathrm{~N}_{3} \mathrm{O} 270.1606$, found 270.1606 .

6fa, yellow solid (27.9 mg, 48\% yield). ${ }^{1} \mathrm{H}$ NMR (400 MHz, $\left.\mathrm{CDCl}_{3}\right): \delta=8.43(\mathrm{~d}, J=4.7 \mathrm{~Hz}, 2 \mathrm{H}), 7.63(\mathrm{~d}, J=2.0 \mathrm{~Hz}, 1 \mathrm{H}), 7.06$ (d, $J=8.2 \mathrm{~Hz}, 1 \mathrm{H}), 6.98(\mathrm{dd}, J=8.2,2.0 \mathrm{~Hz}, 1 \mathrm{H}), 6.75(\mathrm{t}, J=4.7$ $\mathrm{Hz}, 1 \mathrm{H}), 6.37$ (t, $J=3.5 \mathrm{~Hz}, 1 \mathrm{H}), 3.74-3.61(\mathrm{~m}, 2 \mathrm{H}), 3.00-2.89$ (m, 1H), 2.74-2.66 (m, 1H), 2.17-2.04 (m, 2H), $1.14(\mathrm{t}, J=7.0$ $\mathrm{Hz}, 3 \mathrm{H}) .{ }^{13} \mathrm{C}$ NMR $\left(100 \mathrm{MHz}, \mathrm{CDCl}_{3}\right): \delta=161.2,157.9,137.9$, 130.7, 129.8, 128.2, 124.1, 123.6, 113.6, 81.8, 62.8, 28.4, 22.62, 15.3. HRMS: $[\mathrm{M}+\mathrm{H}]^{+}$calculated for $\mathrm{C}_{15} \mathrm{H}_{17} \mathrm{ClN}_{3} \mathrm{O} 290.1060$, found 290.1061 .

6ga, yellow oil (15.7 mg, 29\% yield). ${ }^{1} \mathrm{H}$ NMR (400 MHz, $\mathrm{CDCl}_{3}$ ): $\delta=8.41(\mathrm{~d}, J=4.8 \mathrm{~Hz}, 2 \mathrm{H}), 7.07(\mathrm{dd}, J=7.8,5.1 \mathrm{~Hz}, 1 \mathrm{H})$, $7.00-6.93(\mathrm{~m}, 2 \mathrm{H}), 6.72(\mathrm{t}, J=4.8 \mathrm{~Hz}, 1 \mathrm{H}), 6.33(\mathrm{dd}, J=5.2,3.9$ $\mathrm{Hz}, 1 \mathrm{H}$ ), 3.74 (q, $J=7.1 \mathrm{~Hz}, 2 \mathrm{H}), 2.90-2.81(\mathrm{~m}, 1 \mathrm{H}), 2.72-2.62$ (m, 1H), 2.36-2.27 (m, 1H), 2.06-1.98 (m, 1H), 1.15 (t, $J=7.1$ $\mathrm{Hz}, 3 \mathrm{H}) .{ }^{13} \mathrm{C}$ NMR $\left(100 \mathrm{MHz}, \mathrm{CDCl}_{3}\right): \delta=161.0,157.9,157.2$ (d, $\left.J_{\mathrm{C}-\mathrm{F}}=248.4 \mathrm{~Hz}\right), 134.9,125.83\left(\mathrm{~d}, J_{\mathrm{C}-\mathrm{F}}=11.0 \mathrm{~Hz}\right), 125.3\left(\mathrm{~d}, J_{\mathrm{C}-\mathrm{F}}=\right.$ $8.3 \mathrm{~Hz}), 123.6\left(\mathrm{~d}, J_{\mathrm{C}-\mathrm{F}}=3.1 \mathrm{~Hz}\right), 113.5\left(\mathrm{~d}, J_{\mathrm{C}-\mathrm{F}}=20.2 \mathrm{~Hz}\right), 113.2$, 83.2, 62.7, 30.3, 23.6, 15.3. HRMS: $[\mathrm{M}+\mathrm{H}]^{+}$calculated for $\mathrm{C}_{15} \mathrm{H}_{17} \mathrm{FN}_{3} \mathrm{O} 274.1356$, found 274.1354 .

6ha, brown solid (38.2 mg, 63\% yield). ${ }^{1} \mathrm{H}$ NMR (400 MHz, $\left.\mathrm{CDCl}_{3}\right): \delta=8.41(\mathrm{~d}, J=4.6 \mathrm{~Hz}, 2 \mathrm{H}), 8.05(\mathrm{~s}, 1 \mathrm{H}), 7.72(\mathrm{~d}, J=5.6$ $\mathrm{Hz}, 2 \mathrm{H}), 7.63(\mathrm{~s}, 1 \mathrm{H}), 7.38-7.31(\mathrm{~m}, 2 \mathrm{H}), 6.71(\mathrm{t}, J=4.6 \mathrm{~Hz}, 1 \mathrm{H})$, $6.50(\mathrm{t}, J=4.4 \mathrm{~Hz}, 1 \mathrm{H}), 3.75-3.65(\mathrm{~m}, 2 \mathrm{H}), 3.12-3.01(\mathrm{~m}, 1 \mathrm{H})$, 2.90-2.79 (m, 1H), 2.42-2.32 (m, 1H), 2.15-2.05 (m, 1H), 1.11 $(\mathrm{t}, J=7.0 \mathrm{~Hz}, 3 \mathrm{H}) .{ }^{13} \mathrm{C}$ NMR $\left(100 \mathrm{MHz}, \mathrm{CDCl}_{3}\right): \delta=161.9,157.9$, 135.8, 132.4, 131.6, 131.1, 127.4, 127.1, 126.3, 125.1, 124.9, 122.2, 113.1, 82.2, 62.6, 30.1, 24.1, 15.4. HRMS: [M+H]+ calculated for $\mathrm{C}_{19} \mathrm{H}_{20} \mathrm{~N}_{3} \mathrm{O} 306.1606$, found 306.1609 .

6ab, yellow solid (39.7 mg, 82\% yield). ${ }^{1} \mathrm{H}$ NMR (400 MHz,
$\left.\mathrm{CDCl}_{3}\right): \delta=8.42(\mathrm{~d}, J=4.6 \mathrm{~Hz}, 2 \mathrm{H}), 7.59(\mathrm{~d}, J=8.0 \mathrm{~Hz}, 1 \mathrm{H})$, $7.20-7.11(\mathrm{~m}, 2 \mathrm{H}), 7.03(\mathrm{t}, J=7.4 \mathrm{~Hz}, 1 \mathrm{H}), 6.71(\mathrm{t}, J=4.7 \mathrm{~Hz}$, $1 \mathrm{H}), 6.30(\mathrm{t}, J=3.7 \mathrm{~Hz}, 1 \mathrm{H}), 3.42(\mathrm{~s}, 3 \mathrm{H}), 2.98-2.87(\mathrm{~m}, 1 \mathrm{H})$, 2.78-2.65 (m, 1H), 2.22-2.07 (m, 2H). ${ }^{13} \mathrm{C}$ NMR (100 MHz, $\left.\mathrm{CDCl}_{3}\right): \delta=161.6,157.8,136.8,130.2,128.7,125.6,124.6$, 123.9, 113.2, 83.6, 55.0, 28.8, 23.2. HRMS: $[\mathrm{M}+\mathrm{H}]^{+}$calculated for $\mathrm{C}_{14} \mathrm{H}_{16} \mathrm{~N}_{3} \mathrm{O} 242.1293$, found 242.1296 .

6ac, yellow solid (43.0 mg, 80\% yield). ${ }^{1} \mathrm{H}$ NMR (400 MHz, $\left.\mathrm{CDCl}_{3}\right): \delta=8.39(\mathrm{~d}, J=4.7 \mathrm{~Hz}, 2 \mathrm{H}), 7.54(\mathrm{~d}, J=8.3 \mathrm{~Hz}, 1 \mathrm{H})$, $7.18-7.11(\mathrm{~m}, 2 \mathrm{H}), 7.02(\mathrm{t}, J=7.3 \mathrm{~Hz}, 1 \mathrm{H}), 6.68(\mathrm{t}, J=4.7 \mathrm{~Hz}$, $1 \mathrm{H}), 6.47(\mathrm{t}, J=3.6 \mathrm{~Hz}, 1 \mathrm{H}), 4.02(\mathrm{dt}, J=12.3,6.1 \mathrm{~Hz}, 1 \mathrm{H})$, $3.01-2.90(\mathrm{~m}, 1 \mathrm{H}), 2.76-2.67(\mathrm{~m}, 1 \mathrm{H}), 2.19-2.02(\mathrm{~m}, 2 \mathrm{H}), 1.26$ $(\mathrm{d}, J=6.0 \mathrm{~Hz}, 3 \mathrm{H}), 0.94(\mathrm{~d}, J=6.3 \mathrm{~Hz}, 3 \mathrm{H}) .{ }^{13} \mathrm{C} \mathrm{NMR}(100 \mathrm{MHz}$, $\mathrm{CDCl}_{3}$ ): $\delta=161.5,157.8,137.0,130.4,128.7,125.3,124.8$, $123.7,112.9,80.2,67.7,29.4,23.2,23.2,22.1$. HRMS: $[\mathrm{M}+\mathrm{H}]^{+}$ calculated for $\mathrm{C}_{16} \mathrm{H}_{20} \mathrm{~N}_{3} \mathrm{O} 270.1606$, found 270.1605 .

6ad, Yellow oil (24.1 mg, 53\% yield). ${ }^{1} \mathrm{H}$ NMR (400 MHz, $\left.\mathrm{CDCl}_{3}\right): \delta=8.37(\mathrm{~d}, J=3.7 \mathrm{~Hz}, 2 \mathrm{H}), 7.55(\mathrm{~d}, J=8.1 \mathrm{~Hz}, 1 \mathrm{H})$, 7.14-7.06 (m, 2H), $6.93(\mathrm{t}, J=7.3 \mathrm{~Hz}, 1 \mathrm{H}), 6.71(\mathrm{t}, J=4.5 \mathrm{~Hz}$, $1 \mathrm{H}), 5.80(\mathrm{t}, J=7.5 \mathrm{~Hz}, 1 \mathrm{H}), 5.5-5.0($ br s, $1 \mathrm{H}), 2.61-2.47(\mathrm{~m}$, $2 \mathrm{H}), 2.46-2.29(\mathrm{~m}, 1 \mathrm{H}), 1.85-1.73(\mathrm{~m}, 1 \mathrm{H}) .{ }^{13} \mathrm{C}$ NMR $(100 \mathrm{MHz}$, $\left.\mathrm{CDCl}_{3}\right): \delta=161.8,157.9,137.4,133.0,127.3,126.2,123.6$, 123.2, 113.4, 80.7, 32.1, 25.4. HRMS: $[\mathrm{M}+\mathrm{H}]^{+}$calculated for $\mathrm{C}_{13} \mathrm{H}_{14} \mathrm{~N}_{3} \mathrm{O} 228.1137$, found 228.1137 .

6ae, yellow oil (20.7 mg, 43\% yield). ${ }^{1} \mathrm{H}$ NMR (400 MHz, $\left.\mathrm{CDCl}_{3}\right): \delta=8.45(\mathrm{~d}, J=4.8 \mathrm{~Hz}, 2 \mathrm{H}), 7.42(\mathrm{~s}, 1 \mathrm{H}), 7.04(\mathrm{~d}, J=7.6$ $\mathrm{Hz}, 1 \mathrm{H}), 6.83(\mathrm{~d}, J=7.6 \mathrm{~Hz}, 1 \mathrm{H}), 6.78(\mathrm{t}, J=4.8 \mathrm{~Hz}, 1 \mathrm{H})$, 5.88-5.81 (m, 1H), $5.28(\mathrm{~d}, J=2.6 \mathrm{~Hz}, 1 \mathrm{H}), 2.62-2.53(\mathrm{~m}, 2 \mathrm{H})$, 2.51-2.46 (m, 1H), 2.32 (s, 3H), 1.87-1.78 (m, 1H). ${ }^{13} \mathrm{C}$ NMR $\left(100 \mathrm{MHz}, \mathrm{CDCl}_{3}\right): \delta=161.9,157.9,137.2,135.8,130.2,127.1$, 124.6, 123.7, 113.3, 80.8, 32.3, 25.0, 21.6. HRMS: $[\mathrm{M}+\mathrm{H}]^{+}$calculated for $\mathrm{C}_{14} \mathrm{H}_{16} \mathrm{~N}_{3} \mathrm{O} 242.1293$, found 242.1296.

\section{Results and discussion}

Quaternary ammonium salts, which served as pivotal intermediates of many natural products [49-52], have attracted increasing attention. Among them, isoquinolinium, pyridinium, quinolizinium and cinnolinium salts have been accessed via $\mathrm{Rh}(\mathrm{III})$-catalyzed C-H activation [53-56]. However, synthesis of quinoliniums has been rather limited. Cheng et al. [57] reported $\mathrm{Cu}(\mathrm{II})$-catalyzed coupling of benzylic azides with alkenes for the synthesis of quinoliniums via a radical processe. We focused on a Rh(III)-catalyzed approach, and we noted that the intermediate $\mathbf{D}$ could be oxidized to quinolinium salts. Thus, we set out to explore the oxidative annulation of $N$-pyridylaniline (1a) and enone. Initially, reaction of 1a, EVK (2a), HOPiv $(0.2$ mmol) and a stoichiometric amount of $\mathrm{AgBF}_{4}$ in the presence of [Cp* $\left.\mathrm{RhCl}_{2}\right]_{2}$ (2.5 mol\%) in EtOH afforded quinolinium salt 3aa in $26 \%$ isolated yield. Further screening of various solvents such as $\mathrm{MeOH}$, TFE, ${ }^{t} \mathrm{AmylOH}$ and ${ }^{t} \mathrm{BuOH}$ gaves $\mathrm{MeOH}$ as the optimal one, and further increasing the reaction temperature to $120{ }^{\circ} \mathrm{C}$ led to a yield of $85 \%$.

With optimal conditions in hand, we next examined the scope and generality of this coupling reaction (Scheme 3). Various substituted vinyl ketone, such as alkyl (3aa, 3ab), aryl (3ac), and internal enones (3ad, 3ae) readily coupled with 1a 


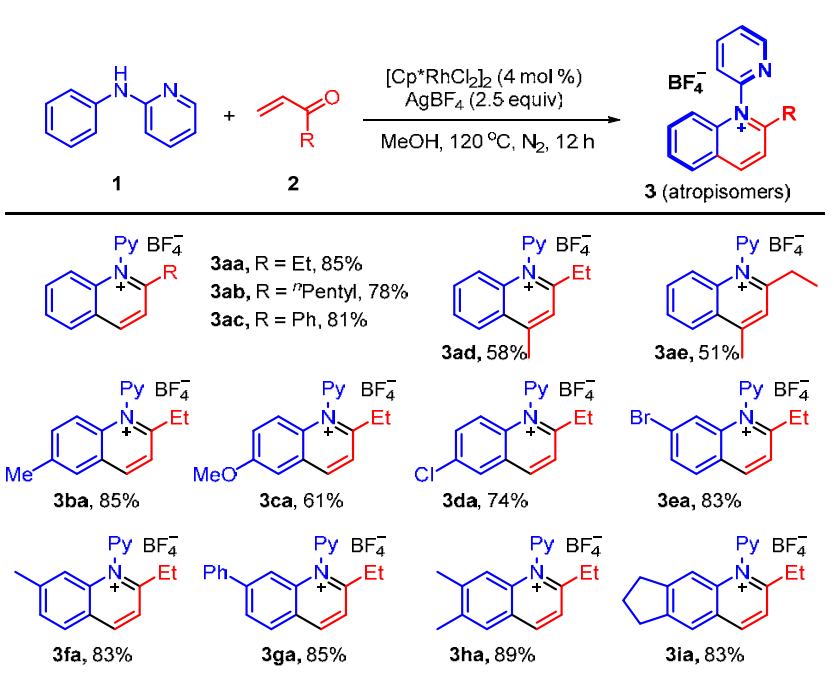

Scheme 3. Coupling of $N$-(2-pyridyl)anilines with enones. Reactions were carried out using $\left[\mathrm{Cp}^{*} \mathrm{RhCl}_{2}\right]_{2}$ (4 mol\%), $\mathrm{AgBF}_{4}$ (2.5 equiv), aniline $(0.2 \mathrm{mmol})$ and enone $(0.4 \mathrm{mmol})$ in methanol $(3.0 \mathrm{~mL})$ at $120^{\circ} \mathrm{C}$ under $\mathrm{N}_{2}$ for $12 \mathrm{~h}$. Isolated yield after column chromatography.

under the standard conditions to afford the annulation products in 51\%-85\% yields. Electron-donating groups, halogen groups, and phenyl at the para/meta position of the benzene ring are also tolerated (61\%-89\%, 3ba-3ga). Furthermore, di-substituted arene $(83 \%-89 \%$, 3ha-3ia) also gave good yields.

Encouraged by these initial findings, we sought to further define the scope of oxidative coupling reaction using acroleins. In this system, the olefin-forming $\beta$-hydrogen elimination needs to be suppressed [34]. In fact, this has been realized in C-H activation systems using highly electrophilic olefins such as acrolein, simple enones, and $\mathrm{CF}_{3}$-substituted enones as a coupling partner, possibly via formation of a stable $\eta^{3}$-enolate species [58-63]. Our initial Rh(III)-catalyzed coupling of $N$-(2-pyridyl)aniline with acrolein using a stoichiometric amount of $\mathrm{Cu}(\mathrm{II})$ oxidant in EtOH afforded the desired dihydroquinolone (4af) in $92 \%$ yield. Of note, during the optimization of this system, a yield of $25 \%$ was also realized even in the absence of any external oxidant, which might indicate either hydrogen-releasing coupling or TH with acrolein being a sacrificial hydrogen acceptor (vida infra) [64-67]. To avoid using a stoichiometric amount of copper(II), different solvents and acid additives were then evaluated. Acetone solvent and HOAc additive turned out to be the optimal choice, and a yield of $89 \%$ was realized when the acrolein was used in two equiv in the presence of a catalytic amount of $\mathrm{Cu}(\mathrm{OAc})_{2}$. In contrast, introduction of $\mathrm{PhNO}_{2}$ [68] or air as co-oxidant all led to reduced yield, and the yield also significantly dropped when acrolein was only provided in 1.1 equiv. It should be noted that although oxidative synthesis of dihydroquinolones has been realized using oxygen as a terminal oxidant under different conditions [44], TH oxidation has not been reported.

Following the optimized conditions, various substituted $N$-(2-pyridyl)anilines were then evaluated to establish the reaction scope (Scheme 4). $N$-Pyridylanilines bearing both electron-donating and -withdrawing groups at the para position all

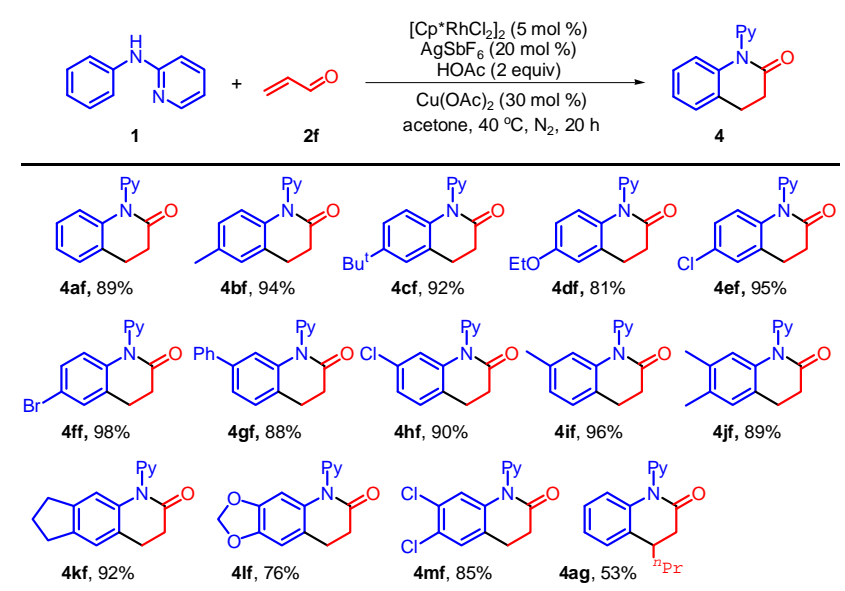

Scheme 4. Scope of oxidative coupling of $N$-(2-pyridyl)anilines with acrolein. Reactions were carried out under conditions: $\left[\mathrm{Cp}^{*} \mathrm{RhCl}_{2}\right]_{2}(5$ mol\%), $\mathrm{AgSbF}_{6}(20 \mathrm{~mol} \%), \mathrm{HOPiv}$ ( 2 equiv), $\mathrm{Cu}(\mathrm{OAc})_{2}$ ( 0.3 equiv), aniline $(0.2 \mathrm{mmol})$, and acrolein $(0.4 \mathrm{mmol})$ in acetone $(3.0 \mathrm{~mL})$ at $40{ }^{\circ} \mathrm{C}$ under the $\mathrm{N}_{2}$ for $20 \mathrm{~h}$. Isolated yield after column chromatography.

coupled in good to excellent yields (4af-4ff) under standard conditions. The $\mathrm{C}-\mathrm{H}$ activation occurred at the less hindered ortho site for anilines bearing a meta substituent (4gf-4if). Disubstituted anilines also coupled in moderate to excellent yields with excellent site-selectivity (4jf-4mf). The acrolein substrate was further extended to 3-propylacrolein, which coupled in moderate yield with $\mathrm{N}$-2-pyridylaniline to give $\mathbf{4 a g}$ that bears a stereogenic center, indicating tolerance of steric hindrance.

To better define the reaction scope, we next explored the coupling of the analogous $\mathrm{N}$-pyrimidylanilines (Scheme 5). Unexpectedly, a totally different outcome was observed. By using ethanol as a solvent, a cyclic hemiaminal ether [69-74]

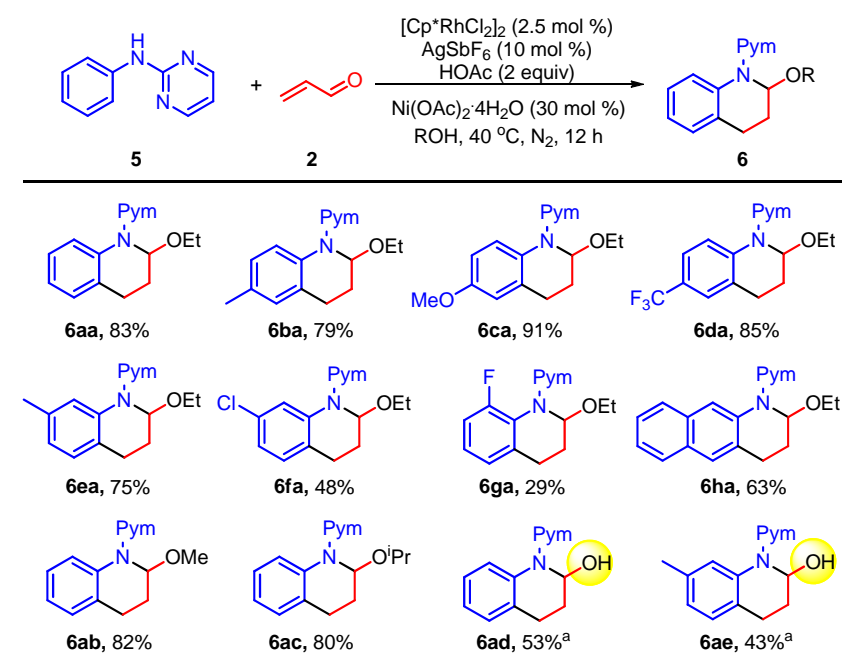

Scheme 5. Scope of redox-neutral coupling of $N$-(2-pyrimidyl)anilines and acrolein. Reactions were carried out using $\left[\mathrm{Cp}^{*} \mathrm{RhCl}_{2}\right]_{2}(2.5 \mathrm{~mol} \%)$, $\mathrm{AgSbF}_{6}(10 \mathrm{~mol} \%), \mathrm{HOAc}$ (2 equiv), $\mathrm{Ni}(\mathrm{OAc})_{2} \cdot 4 \mathrm{H}_{2} \mathrm{O}$ (30 mol\%), aniline $(0.2 \mathrm{mmol})$, and acrolein $(0.4 \mathrm{mmol})$ in EtOH $(3.0 \mathrm{~mL})$ at $40{ }^{\circ} \mathrm{C}$ under the $\mathrm{N}_{2}$ for $12 \mathrm{~h}$. Isolated yield after column chromatography. a [Cp* $\mathrm{RhCl}_{2}$ ] (2.5 mol\%), AgSbF 6 (10 mol\%), HOPiv (1 equiv), aniline $(0.2 \mathrm{mmol})$, and acrolein $(0.4 \mathrm{mmol})$ in 1,4 -dioxane $(3.0 \mathrm{~mL})$ at $40{ }^{\circ} \mathrm{C}$ under $\mathrm{N}_{2}$ for $20 \mathrm{~h}$. 
(6aa) was isolated under various conditions in the presence of absence of any $\mathrm{Cu}(\mathrm{II})$ oxidant. After extensive optimization studies, the yield was maximized (83\%) when a catalytic amount of $\mathrm{Ni}(\mathrm{OAc})_{2}$ was introduced, which may function as a mild Lewis acid to promote elimination of water for iminium formation. The scope of this reaction has been briefly explored. Such anilines bearing various electron-donating and -withdrawing substituents at the para, meta, and ortho positions all coupled in mild to good yields in ethanol (6aa-6ha). Other common alcohols such as $\mathrm{MeOH}$ and $i$-PrOH were also applicable, affording hemiaminal ethers $\mathbf{6} \mathbf{a b}$ and $\mathbf{6 a c}$ in high yields. When the solvent was replaced by a non-nucleophilic solvent such as 1,4-dioxane, the corresponding cyclic hemiaminals $6 \mathbf{a d}$ and $6 \mathbf{a e}$ were isolated in moderate yield due to instability.

In this coupling system (Scheme 5), the chemoselectivity is likely dictated by the directing group. In fact, Chang et al. [75] have demonstrated that in Ir(III) catalysis, the DG significantly affected chemoselectivity by modifying the conformation and electronic effects of the intermediate. We rationalized that a 2-pyrimidyl group renders a more electron-poor metal center than the $N$-pyridyl, which will disfavor the dissociation of an anionic ligand to make a vacant site for $\beta$-H elimination. Consequently, it may favor the competitive formation of the iminium species which is nucleophilically trapped by the alcohol to give the hemiaminal ether.
Several experiments have been performed to explore the mechanism (Scheme 6). In the oxidative synthesis of 4 af, we have verified that none of acrylic acid, acrylate ester, or allyl alcohol was an active intermediate (Scheme 6(a)). This suggests that aldehyde is an essential functionality in this system. In the meantime, we managed to clarify the source of the oxidant in the coupling of 1a and acrolein ( 2 equiv) in acetone- $d_{6}$ in the absence of $\mathrm{Cu}(\mathrm{OAc})_{2}$ (Scheme $6(\mathrm{~b})$ ). ${ }^{1} \mathrm{H}$ NMR analysis of this reaction in a $J$-Young tube revealed that the coupled product 4af, propanal, allyl alcohol, and $\mathrm{CD}_{3} \mathrm{CH}(\mathrm{OH}) \mathrm{CD}_{3}$ were generated in 3.5:1:1:0.7 ratio, indicating that acrolein is the major source of sacrificial oxidant. Some experiments have been performed to clarify the reaction intermediate in synthesis of quinolinium salt 3aa (Schemes 6(c)). The hydroarylation-generated ketone $\mathbf{8}$ proved to be an intermediate during the formation of 3aa. In addition, treatment of dihydroquinoline 7aa with $\mathrm{AgBF}_{4}$ also led to formation of product 3aa in good yield as a result of formal abstraction of the $\alpha-\mathrm{H}$ as a hydride. This mechanism of salt synthesis stays complementary to those previously reported that rely on $\mathrm{C}-\mathrm{N}$ reductive elimination [76]. Direct comparisons of Py and Pym, DGs have been made in oxidative and redox-neutral coupling systems by replacing each other in the original optimal conditions (Scheme 6(d)). In the oxidative coupling of 1a with acrolein (2f), simply switching the Py to Pym resulting no formation of the 4af. Instead, the hemiaminal product $6 \mathbf{a a}$ was isolated in $40 \%$ yield.

(a) Control experiments

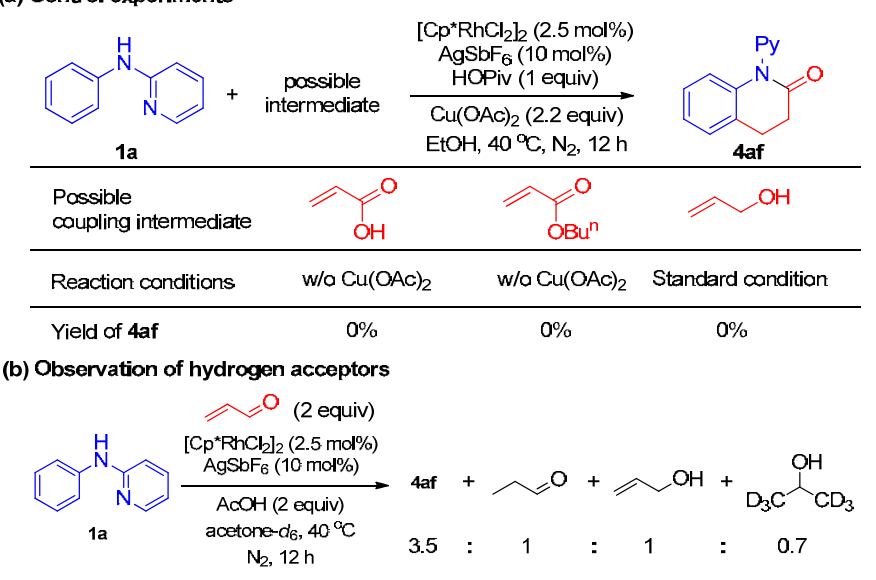

(c) Intermediates in quinolinium synthesis

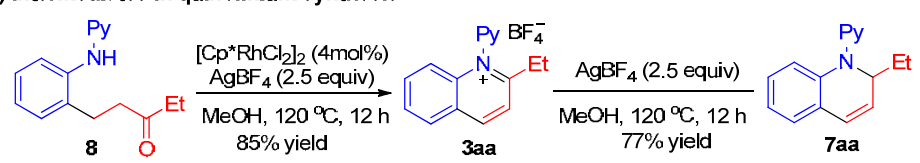

(d) Comarisions of the directing group

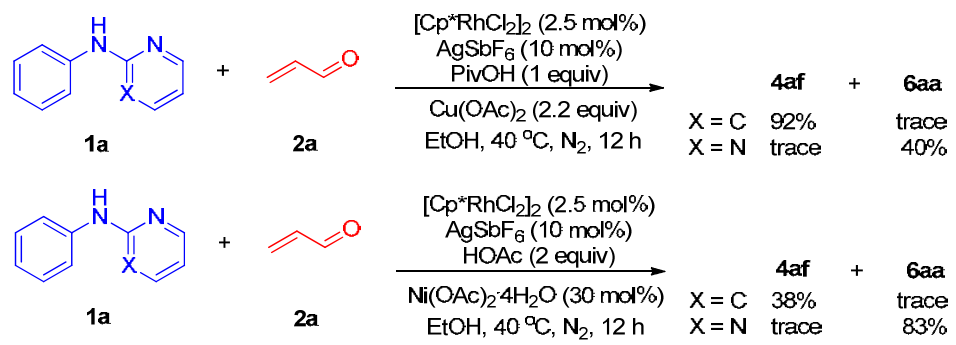

Scheme 6. Mechanistic studies. 
Under redox-neutral conditions, switching the Pym to Py only afforded 4 af in 38\% yield, indicating that the chemoselectivity was directly dependent on the nature of the DG.

\section{Conclusions}

In summary, we have demonstrated rhodium(III)-catalyzed chemo- and redox-selective annulation of $\mathrm{N}$-substituted anilines with acroleins/ketones, which allowed access to structurally diverse heterocycles. The selectivity for the coupling of acrolein was found to be controlled by the $N$-directing group. The TH-oxidative coupling of $N$-pyridylaniline afforded dihydroquinolones with the acrolein being a major hydrogen acceptor, while the coupling of $N$-pyrimidylaniline in an alcoholic solvent is redox-neutral. In addition, oxidative annulation between $N$-pyridylaniline and enone delivered a quinolinium salt. The redox diversity in heterocycle synthesis may find applications in development of new $\mathrm{C}-\mathrm{H}$ activation systems.

\section{References}

[1] J. C. Lewis, R. G. Bergman, J. A. Ellman, Acc. Chem. Res., 2008, 41, 1013-1025.

[2] L. Ackermann, Chem. Rev., 2011, 111, 1315-1345.

[3] D. Y. K. Chen, S. W. Youn, Chem. Eur. J., 2012, 18, 9452-9474.

[4] R. Y. Zhu, M. E. Farmer, Y. Q. Chen, J. Q. Yu, Angew. Chem. Int. Ed., 2016, 55, 10578-10599.

[5] T. Gensch, M. N. Hopkinson, F. Glorius, J. Wencel-Delord, Chem. Soc. Rev., 2016, 45, 2900-2936.

[6] Y. Wei, P. Hu, M. Zhang, W. P. Su, Chem. Rev., 2017, 117, 8864-8907.

[7] Y. Park, Y. Kim, S. Chang, Chem. Rev., 2017, 117, 9247-9301.
[8] C. G. Newton, S. G. Wang, C. C. Oliveira, N. Cramer, Chem. Rev., 2017, 117, 8908-8976.

[9] J. R. Hummel, J. A. Boerth, A. J. Ellman, Chem. Rev., 2017, 117, 9163-9227.

[10] Y. D. Yang, J. B. Lan, J. S. You, Chem. Rev., 2017, 117, 8787-8863.

[11] T. Piou, T. Rovis, Acc. Chem. Res., 2018, 51, 170-180.

[12] S. R. Neufeldt, M. S. Sanford, Acc. Chem. Res., 2012, 45, 936-946.

[13] J. J. Mousseau, A. B. Charette, Acc. Chem. Res., 2013, 46, 412-424.

[14] T. Iwai, M. Sawamura, ACS. Catal., 2015, 5, 5031-5040.

[15] J. Park, S. Chang, Chem. Asian J., 2018, 13, 1089-1102.

[16] O. Eisenstein, J. Milani, R. N. Perutz, Chem. Rev., 2017, 117, 8710-8753.

[17] T. Yoshino, S. Matsunaga, Adv. Synth. Catal., 2017, 359, $1245-1262$.

[18] D. Vidal, G. Olivo, M. Costas, Chem. Eur. J., 2018, 24, 5042-5054.

[19] X. C. Cambeiro, T. C. Boorman, P. F. Lu, I. Larrosa, Angew. Chem. Int. Ed., 2013, 52, 1781-1784.

[20] C. Huang, J. Wu, C. J. Song, R. Ding, Y. Qiao, H. W. Hou, J. B. Chang, Y. T. Fan, Chem. Commun., 2015, 51, 10353-10356.

[21] K. H. He, W. D. Zhang, M. Y. Yang, K. L. Tang, M. N. Qu, Y. S. Ding, Y. Li, Org. Lett., 2016, 18, 2840-2843.

[22] S. Hammes-Schiffer, ChemPhysChem, 2002, 3, 33-42.

[23] R. H. D. Lyngdoh, H. F. Schaefer III, Acc. Chem. Res., 2009, 42, 563-572.

[24] L. Wang, J. Xiao, Top. Curr. Chem., 2016, 374, 17-71.

[25] D. B. Rice, A. A. Massie, T. A. Jackson, Acc. Chem. Res., 2017, 50, 2706-2717.

[26] T. Satoh, M. Miura, Chem. Eur. J., 2010, 16, 11212-11222.

[27] G. Y. Song, X. W. Li, Acc. Chem. Res., 2015, 48, 1007-1020.

[28] Y. D. Yang, K. Z. Li, Y. Y. Cheng, D. Y. Wan, M. L. Li, J. S. You, Chem. Commun., 2016, 52, 2872-2884.

[29] P. Gandeepan, C. H. Cheng, Chem. Asian. J., 2016, 11, 448-460.

[30] M. Gulias, J. L. Mascarenas, Angew. Chem. Int. Ed., 2016, 55, 11000-11019.

[31] X. F. Wu, Transition Metal-Catalyzed Heterocycle Synthesis via $C-H$

\section{Graphical Abstract}

Chin. J. Catal., 2018, 39: 1782-1791 doi: 10.1016/S1872-2067(18)63134-1

Chemo-selective couplings of anilines and acroleins/enones under substrate control and condition control

Xukai Zhou, Jiaqiong Sun, Xingwei Li*

Dalian Institute of Chemical Physics, Chinese Academy of Sciences; University of Chinese Academy of Sciences

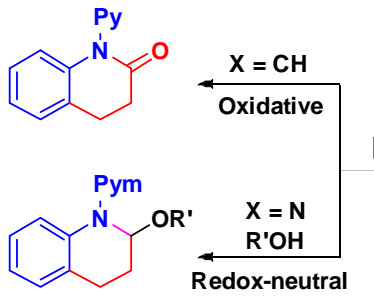

- Selectivity under substrate and condition control

- Acrolein as a hydrogen acceptor

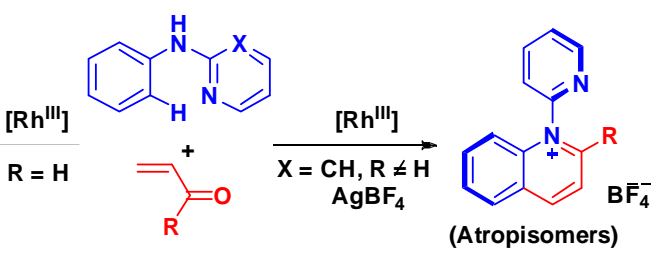

- Divergent synthesis

- 39 Examples in total

$\mathrm{Rh}(\mathrm{III}$ )-catalyzed $\mathrm{C}-\mathrm{H}$ activation of $\mathrm{N}$-protected anilines and chemo-divergent couplings with acroleins/enones have been realized for synthesis of three classes of heterocycles. The oxidative coupling of $N$-pyridylaniline afforded dihydroquinolones with the acrolein being a major hydrogen acceptor. When the directing group was replaced by pyrimidyl in the same system, redox-neutral coupling occurred to afford hemiaminal ethers. Oxidative annulation of $N$-pyridylanilines with enones using $\mathrm{AgBF}_{4}$ oxidant afforded atropisomeric quinolinium salts. 
Activation, Wiley-VCH: Weinheim, 2016.

[32] Y. W. Xu, F. Wang, S. J. Yu, X. W. Li, Chin. J. Catal., 2017, 38, 1390-1398.

[33] X. K. Zhou, S. J. Yu, Z. S. Qi, X. W. Li, Sci. China. Chem., 2015, 58, 1297-1301.

[34] J. L. Chen, G. Y. Song, C. L. Pan, X. W. Li, Org. Lett., 2010, 12, 5426-5429.

[35] J. T. Xia, X. F. Yang, Y. Y. Li, X. W. Li, Org. Lett., 2017, 19, 3243-3246.

[36] G. D. Tang, C. L. Pan, X. W. Li, Org. Chem. Front., 2016, 3, 87-90.

[37] F. X. Zhu, Y. H. Li, Z. C. Wang, X. F. Wu, Adv. Synth. Catal., 2016, 358, 3350-3354.

[38] X. K. Zhou, J. T. Xia, G. F. Zheng, L. H. Kong, X. W. Li, Angew. Chem. Int. Ed., 2018, 57, 6681-6685.

[39] Z. Mazloomi, R. Pretorius, O. Pàmies, M. Albrecht, M. Diéguez, Inorg. Chem., 2017, 56, 11282-11298.

[40] K. M. Waldie, K. R. Flajslik, E. McLoughlin, C. E. D. Chidsey, R. M. Waymouth, J. Am. Chem. Soc., 2017, 139, 738-748.

[41] F. Saleem, G. K. Rao, A. Kumar, G. Mukherjee, A. K. Singh, Organometallics, 2014, 33, 2341-2351.

[42] K. I. Fujita, Y. Takahashi, M. Owaki, K. Yamamoto, R. Yamaguchi, Org. Lett., 2004, 6, 2785-2788.

[43] S. Sharma, E. Park, J. Park, I. S. Kim, Org. Lett., 2012, 14, 906-909.

[44] Z. J. Wu, K. L. Huang, Z. Z. Huang, Org. Biomol. Chem., 2017, 15, 4978-4983.

[45] M. Albrecht, R. H. Crabtree, J. Mata, E. Peris, Chem. Commun., 2002, 32-33.

[46] X. M. Huang, S. G. Xu, Q. T. Tan, M. C. Gao, M. J. Li, B. Xu, Chem. Commun., 2014, 50, 1465-1468.

[47] N. K. Mishra, M. Choi, H. Jo, Y. Oh, S. Sharma, S. H. Han, T. Jeong, S. Han, S. Y. Lee, I. S. Kim, Chem. Commun., 2015, 51, 17229-17232.

[48] S. Chanthamath, S. Takaki, K. Shibatomi, S. Iwasa, Angew. Chem. Int. Ed., 2013, 52, 5818-5821.

[49] J. P. Michael, Nat. Prod. Rep., 2007, 24, 191-222.

[50] J. P. Michael, Nat. Prod. Rep., 2008, 25, 139-165.

[51] L. Y. Wei, A. Brossi, S. L. Morris-Natschke, K. F. Bastow, K. H. Lee, Stud. Nat. Prod. Chem., 2008, $34,3$.

[52] J. P. Michael, The Alkaloids Chemistry Biology, 2016, 75, 1-498.

[53] C. Z. Luo, P. Gandeepan, Y. C. Wu, C. H. Tsai, C. H. Cheng, ACS Catal,, 2015, 5, 4837-4841.
[54] P. Gandeepan, C. H. Cheng, Chem. Asian J., 2016, 11, 448-460.

[55] J. Jayakumar, C. H. Cheng, Chem. Eur. J., 2016, 22, 1800-1804.

[56] N. S. Upadhyay, J. Jayakumar, C. H. Cheng, Chem. Commun., 2017, 53, 2491-2494.

[57] W. C. Chen, P. Gandeepan, C. H. Tsai, C. Z. Luo, P. Rajamalli, C. H. Cheng, RSC Adv., 2016, 6, 63390-63397.

[58] Z. Z. Shi, C. Grohmann, F. Glorius, Angew. Chem. Int. Ed., 2013, 52, 5393-5397.

[59] P. P. Duan, X. Lan, Y. Chen, S. S. Qian, J. J. Li, L. Lu, Y. B. Lu, B. Chen, M. Hong, J. Zhao, Chem. Commun., 2014, 50, 12135-12138.

[60] J. A. Boerth, J. A. Ellman, Chem. Sci, 2016, 7, 1474-1479.

[61] A. B. Weinstein, J. A. Ellman, Org. Lett, 2016, 18, 3294-3297.

[62] T. J. Potter, J. A. Ellman, Org. Lett., 2016, 18, 3838-3841.

[63] B. X. Liu, P. J. Hu, Y. Zhang, Y. Y. Li, D. C. Bai, X. W. Li, Org. Lett, 2017, 19, 5402-5405.

[64] X. W. Li, P. Chen, J. W. Faller, R. H. Crabtree, Organometallics, 2005, $24,4810-4815$.

[65] D. C. Leitch, J. A. Labinger, J. E. Bercaw, Organometallics, 2014, 33, 3353-3365.

[66] M. Morimoto, T. Miura, M. Murakami, Angew. Chem. Int. Ed., 2015, 54, 12659-12663.

[67] W. H. Li, L. Wu, S. S. Li, C. F. Liu, G. T. Zhang, L. Dong, Chem. Eur. J., 2016, 22, 17926-17929.

[68] N. N. Lü, Y. Liu, C. H. Xiong, Z. X. Liu, Y. H. Zhang, Org. Lett., 2017, $19,4640-4643$.

[69] H. Steinhagen, E. J. Corey, Angew. Chem. Int. Ed., 1999, 38, 1928-1931.

[70] C. L. Mathis, B. M. Gist, C. K. Frederickson, K. M. Midkiff, C. C. Marvin, Tetrahedron Lett., 2013, 54, 2101-2104.

[71] M. L. Deb, S. S. Dey, I. Bento, M. T. Barros, C. D. Maycock, Angew. Chem. Int. Ed., 2013, 52, 9791-9795.

[72] A. Modak, U. Dutta, R. Kancherla, S. Maity, M. Bhadra, S. M. Mobin, D. Maiti, Org. Lett., 2014, 16, 2602-2605.

[73] K. L. Chen, B. Gao, Y. G. Shang, J. Y. Du, Q. L. Gu, J. X. Wang, Org. Biomol. Chem., 2017, 15, 8770-8779.

[74] P. Bai, Y. Q. Li, Z. Z. Huang, Org. Lett., 2017, 19, 1374-1377.

[75] J. Kim, S. W. Park, M. H. Baik, S. Chang, J. Am. Chem. Soc., 2015, 137, 13448-13451.

[76] P. Gandeepan, C. H. Cheng, Chem. Asian J., 2016, 18, 448-460.

\title{
底物与条件控制苯胺与丙烯醛/烯酮的化学选择性偶联反应
}

\author{
周旭凯 ${ }^{\mathrm{a}, \mathrm{b}}$, 孙佳琼 ${ }^{\mathrm{a}}$, 李兴伟 ${ }^{\mathrm{a}}$ * \\ a 中国科学院大连化学物理研究所, 辽宁大连 116023 \\ b 中国科学院大学, 北京100049
}

摘要: 金属催化碳氢键活化已经成为制备高附加值有机化合物的一类高效方法, 由于碳氢键广泛存在, 所以对它们进行化 学、区域、立体选择性的活化作为一大挑战已经被人们日益所关注, 然而氧化还原选择性控制的研究十分少见. 通常而言, 有机氧化还原反应定义为得失氢氧原子, 例如消除氢负离子为氧化反应, 而失去一个质子则为中性反应. 在已有的研究中 单独的氧化、还原反应已经被广泛研究, 而且被大量用于医药合成、精细化工品的制备以及各类先进材料的生成. 但是在 同一反应体系下同时调控三种氧化态的研究目前未见报道, 因此发展这类选择性控制的反应十分重要. 我们此前已经实 现了 Rh(III)/Ir(III)催化苯胺和烯酮还原偶联合成四氢喹啉和高烯丙基苯胺两种产物, 同时也能得到中性的1,2-二氢喹啉产 物. 在此工作的基础上, 我们希望能够进一步实现相同反应组分的氧化偶联. 为此, 我们仔细分析取代的苯胺与烯酮的可 能反应路径, 发现可能的关键物种 $\mathbf{G}$ 一含有 $\mathrm{Rh}(\mathrm{III})$ 的六元杂环中间体有望实现这类氧化过程, 当用丙烯醛做底物时, 物种 $\mathbf{G}$ 有可能实现 $\beta$-氢消除得到氧化的二氢喹啉酮和 $\mathrm{Cp} * \mathrm{RhXH}$, 通常 $\mathrm{Cp} * \mathrm{RhXH}$ 很容易发生自身的还原消除得到 $\mathrm{Cp} * \mathrm{Rh}(\mathrm{I})$ 使反应 终止, 但是, 丙烯醛的存在有可能重新活化Cp*RhXH使得催化循环一直进行下去. 另一种情况是中间体 $\mathrm{G}$ 发生质子解然后 
脱水得到亚胺物种, 亚胺很容易被亲核试剂进攻得到中性的氮杂缩醛类产物. 当然, 外加银盐氧化剂还有可能得到另一种 氧化型的喹啉盐.

基于这种思路, 我们发展了 Rh(III)-催化碳氢活化N-取代的苯胺与丙烯醛/烯酮的选择性偶联反应, 反应可以化学选择 性专一地制备三类不同的杂环化合物. 当氮-吡啶基苯胺与丙烯醛反应时, 反应类型为氧化过程, 经历了转移氢化的过程, 其中烯醛为主要的氢受体, 得到二氢喹啉酮产物; 如果定位基换成嘧啶时, 在相似的反应条件下, 反应类型为氧化还原中性 过程, 生成氮杂缩醛醚类产物; 氮-吡啶基苯胺和烯酮反应在 $\mathrm{AgBF}_{4}$ 的氧化作用下同样可以发生氧化反应得到喹啉盐类化合 物. 至此, 我们实现了导向基团对氧化反应和中性反应的控制, 氧化剂的种类对反应路径的改变. 反应的底物范围广泛, 官 能团容忍性好, 我们期待这类氧化还原多样性的杂环合成方法能促进更多新颖反应的发现.

关键词: Rh(III)催化; 化学选择性; 环化; 底物调控; 条件调控

收稿日期: 2018-06-29. 接受日期: 2018-07-05. 出版日期: 2018-11-05.

*通讯联系人. 电话: (0411)84379089; 电子信箱: xwli@dicp.ac.cn

基金来源: 国家自然科学基金(21525208, 21472186); 中国科学院大连化学物理研究所甲醇转化与煤代油专项基金.

本文的电子版全文由Elsevier出版社在ScienceDirect上出版(http://www.sciencedirect.com/science/journal/18722067). 